\title{
Investigating Cell Type Specific Mechanisms Contributing to Acute Oral Toxicity
}

\author{
Pilar Prieto, Rabea Graepel, Kirsten Gerloff, Lara Lamon, Magdalini Sachana, Francesca Pistollato, \\ Laura Gribaldo, Anna Bal-Price and Andrew Worth
}

EU Commission Joint Research Centre (JRC), Ispra, Italy

\begin{abstract}
The replacement of animals in acute systemic toxicity testing remains a considerable challenge. Only animal data are currently accepted by regulators, including data generated by reduction and refinement methods. The development of integrated approaches to testing and assessment (IATA) is hampered by an insufficient understanding of the numerous toxicity pathways that lead to acute systemic toxicity. Therefore, central to our work has been the collection and evaluation of the mechanistic information on eight organs identified as relevant for acute systemic toxicity (nervous system, cardiovascular system, liver, kidney, lung, blood, gastrointestinal system, and immune system). While the nervous and cardiovascular systems are the most frequent targets, no clear relationship emerged between specific mechanisms of target organ toxicity and the level (category) of toxicity. From a list of 114 chemicals with acute oral in vivo and in vitro data, 97 were identified with target organ specific effects, of which 94\% (91/97) were predicted as acutely toxic by the 3 T3 neutral red uptake cytotoxicity assay and 6\% (6/97) as non-toxic. Although specific target organ mechanisms of toxicity could in some cases explain the false negative prediction obtained with the cytotoxicity assay, in general it is difficult to explain in vitro misclassifications only on the basis of mechanistic information. This analysis will help to prioritize the development of adverse outcome pathways for acute oral toxicity, which will support the assessment of chemicals using mechanistically informed IATA.
\end{abstract}

\section{Introduction}

Acute systemic toxicity after oral, dermal, or inhalation exposure requires that the substance becomes bioavailable at the target site and induces lethality through general toxicity or a specific mechanism. This means that kinetic factors, and mainly absorption, are important determinants of toxicity (EURL ECVAM, 2015). In addition, if the damage involves interference with homeostatic mechanisms at the organ system level, non-exposed tissues and vital organs can also be affected (Gennari et al., 2004; Andrew, 2013).

The assessment of acute systemic toxicity is a core component of the safety assessment of substances in the context of EU and international legislations (Hamm et al., 2017). Information requirements vary depending on the type of substance subject to regulation and the region (EU, 2006, 2008, 2009a,b, 2012). The regulatory landscape in the USA was reviewed during two workshops cosponsored by the NTP Interagency Center for the Evaluation of Alternative Toxicological Methods (NICEATM), the People for the Ethical Treatment of Animals (PETA) International Science Consortium Ltd, and the Physicians Committee for Responsible Medicine (PCRM) (Hamm et al., 2017; Clippinger et al., 2018a; Strickland et al., 2018). The relevant information is available at the PETA website ${ }^{1}$. In preclinical drug development, however, these studies are no longer required by default to support first clinical trials in humans (Robinson et al., 2008; ICH, 2009; Chapman et al., 2010).

One of the main uses of acute systemic toxicity data is classification and labelling (Seidle et al., 2010; Graepel et al., 2016; Buesen et al., 2016; Strickland et al., 2018). Within the EU, the CLP (Classification, Labelling and Packaging) Regulation (EU, 2008) is used to classify chemicals on the basis of acute oral toxicity into four toxicity categories (categories 1 to 4 of the United Nations Globally Harmonised System of Classification and La-

1 https://www.piscltd.org.uk/acute-systemic-toxicity/ (accessed 28.06.2018).

Received May 18, 2018: Accepted July 6, 2018; Epub July 12, 2018; (C) The Authors, 2018.

ALTEX 36(1), 39-64. doi:10.14573/altex.1805181

Correspondence: Pilar Prieto, PhD, European Commission, Joint Research Centre,

Directorate F - Health, Consumers and Reference Materials,

Chemical Safety and Alternative Methods Unit,

EURL ECVAM, Ispra, Italy

(pilar.prieto-peraita@ec.europa.eu)
This is an Open Access article distributed under the terms of the Creative Commons Attribution 4.0 International license (http://creativecommons.org/licenses/by/4.0/) which permits unrestricted use, distribution and reproduction in any medium, provided the original work is appropriately cited. 
belling (UN GHS)). While CLP does not require animal testing, the classification criteria are based on data derived from animal tests (conducted, for example, under other pieces of legislation), including reduction and refinement methods for the oral, dermal, and inhalation routes (OECD TGs 402, 403, 420, 423, 425, $433,436)$. Most of the standard in vivo tests use lethality as the endpoint, even though this has been widely criticized both on animal welfare and scientific grounds (Zbinden and Flury-Roversi, 1981; Hoffmann et al., 2010; Prieto et al., 2013a).

Basal cytotoxicity is certainly a key factor in many prevalent toxicological modes-of-action associated with acute health effects. It covers many general mechanisms of toxicity common to most cell types that can lead to organ failure including, for example, disruption of cell membrane structure or function, inhibition of mitochondrial function, disturbance of protein turnover, and disruption of metabolism and energy production (Gennari et al., 2004; NIH, 2009; Andrew, 2013). This is the reason why the utility of in vitro cytotoxicity assays to predict acute oral toxicity has been extensively investigated (Ekwall, 1999; Halle, 2003; NIH, 2006; Prieto et al., 2013a,b). Recently, Vinken and Blaauboer (2017) proposed the application of an adverse outcome pathway (AOP) framework for basal cytotoxicity consisting of three consecutive steps, i.e., initial cell injury, mitochondrial dysfunction, and cell death. The outcome of the basal cytotoxicity was then suggested by the authors as the first step of a tiered strategy aimed to evaluate the toxicity of new chemical entities. Further, in a second step, more specific types of toxicity could be evaluated.

One of the better known and standardized in vitro methods for basal cytotoxicity is the 3T3 Neutral Red Uptake (NRU) assay (DB-ALM protocol 1392; Stokes et al., 2008). The use of data from the NRU cytotoxicity assay within a Weight-of-Evidence (WoE) assessment is one of the choices for adapting the standard information requirements for acute oral toxicity, as described in the last update of the ECHA's guidance on Information Requirements and Chemical Safety Assessment. This WoE adaptation proposed by ECHA applies primarily to low toxicity substances (i.e., those that are not to be classified for acute toxicity) and it is based on an in-depth analysis of the REACH database (Gissi et al., 2017; ECHA, 2017). Nevertheless, the limitations of the in vitro cytotoxicity assay, such as the lack of metabolic competence of $3 \mathrm{~T} 3$ cells and difficulty to capture specific mechanisms of action relating to interaction with specific molecular targets in certain tissues, need to be considered when building a WoE case for the purposes of REACH (Buesen et al., 2018; Gissi et al., 2018).

In addition to the assessment of basal cytotoxicity, it is also important to identify cell types and in vitro endpoints that are indicative of cell-type specific toxicities, with a view to incorporating such endpoints into integrated approaches to testing and assessment (IATA), as proposed in the EURL ECVAM strategy to replace, reduce, and refine the use of animals in the assessment of acute mammalian systemic toxicity (EURL ECVAM, 2014). As defined by the OECD, IATA are pragmatic, science-based approaches for chemical hazard or risk characterization that rely on an integrated analysis of existing information in a WoE assessment coupled with the generation of new information, if required (OECD, 2016a). An iterative approach that preferably relies on mechanistic information or available AOPs is followed to answer a defined question in a specific regulatory context, taking into account the acceptable level of uncertainty associated with the decision making (OECD, 2016a; Sachana and Leinala, 2017). The importance of understanding the mechanisms of acute toxicity was further recognized during an international workshop in which a group of experts discussed alternative approaches for identifying acute systemic toxicity (Hamm et al., 2017; Clippinger et al., 2018a). A better theoretical and mechanistic understanding of acute systemic toxicity would be useful to developers of test methods and other predictive tools as well as to validation and regulatory bodies.

Mechanisms involved in cellular failure and susceptible functions compromised in organ failure were discussed at an ECVAM workshop on strategies to replace in vivo acute systemic toxicity testing (Gennari et al., 2004). Several fundamental cellular processes common to many organ systems were identified, including energy production and metabolism (mitochondrial function and glycolysis), transportation of molecules, membrane integrity and secretion of molecules (enzymes, proteins, hormones, neurotransmitters). A number of key events associated with acute human poisoning were further identified in an ICCVAM/ECVAM/ JaCVAM workshop on acute chemical safety testing (NIH, 2009) and it was agreed that mechanistic information could be used to develop more predictive in vitro test methods. A report, commissioned by the US Department of Defense, lists several of the cellular targets or molecular targets that are often associated with the acute lethal or debilitating effects of chemicals. This includes changes in neurotransmission function, altered ion flow, increased permeability of cellular membranes, altered bioenergetics, altered oxygen transport, oxidative stress and reactive oxygen species (ROS) formation, damage to DNA and subcellular systems, and immune-mediated effects (NRC, 2015). Hamm et al. (2017) and Clippinger et al. (2018b) have also reported some of the known mechanisms involved in acute systemic toxicity as part of ongoing activities in the US.

However, despite all the efforts made over the past 20 years in the area of acute systemic toxicity, relevant AOPs, mechanistically informed alternative methods, and IATA for acute systemic toxicity have not been adequately developed. This is partially due to the lack of a complete mechanistic understanding of the key acute toxicity pathways in humans specific for different cell types (e.g., neuronal, cardiac, liver, or kidney).

This study describes the analysis of mechanistic information collected on eight potential organs (i.e., nervous system, cardiovascular system, liver, kidney, lung, blood, gastrointestinal system (GI), and immune system) identified as relevant for acute systemic toxicity and using a set of chemicals inducing acute toxicity after oral exposure. This work will support the development of AOPs and IATA in the area of acute systemic toxicity,

2 https://ecvam-dbalm.jrc.ec.europa.eu/ (accessed 28.06.2018). 
and will inform the development and application of mechanistically relevant new approach methodologies.

\section{Materials and methods}

\section{Collection of mechanistic information}

Information was collected on the eight potential target organs identified as relevant for acute systemic toxicity during the ECVAM workshop on acute systemic toxicity (Gennari et al., 2004): liver, blood, kidney, cardiovascular system, central and peripheral nervous system (CNS/PNS), lung, immune system, and GI. In safety pharmacology studies, the cardiovascular, respiratory, and central nervous systems are assessed in a core battery since they are considered vital organs or systems, the functions of which are acutely critical for life (ICH, 2000).

In order to approach the ambitious task of mapping mechanisms specific for these potential target organs, a three-step approach was taken to identify the potential pathways of target organ toxicity.

1. Based on a literature review using *target organ* and *acute toxicity* and *mechanism* as key words, commonly recognized pathways of toxicity were identified for each target organ/system. Information was derived from published literature, toxicology handbooks, short descriptions of reference compounds used in the EU FP6 project ACuteTox ${ }^{3}$, and internet databases $\left(\mathrm{HSDB}^{4}, \mathrm{INCHEM}^{5}, \mathrm{PubChem}^{6}, \mathrm{PubMed}^{7}\right.$, Scopus $^{8}$, Google Scholar ${ }^{9}$ ). The pathways were then organized and visualized according to the target organ/system, the cell type, the effect, and the mechanism. In this context, the effect refers to any adverse reaction that could be observed or measured (in vivo) and the mechanism refers to the molecular or cellular process that is interrupted by chemical stressors and leads to the observed adverse effect.

2. In the second phase, the "completeness" of the theoretical pathways of toxicity that were developed in phase 1 was probed. In order to do so, we consulted the in-house database and selected chemicals that were shown to be acutely toxic. For these chemicals, a thorough literature search was conducted to identify the target organ and mechanism of toxicity, searching first for *chemical* AND *acute toxicity* and $*$ mechanism*, followed by $*$ chemical $*$ AND *target organ*. These mechanisms were then added into the generated "maps" if they were not already present. Chemicals for which both in vivo acute oral toxicity data and in vitro cytotoxicity data were available were selected. On the basis of reference in vivo oral $\mathrm{LD}_{50}$ data and of the $2000 \mathrm{mg} / \mathrm{kg}$ body weight threshold introduced by the CLP Regulation, all compounds with an acute $\mathrm{LD}_{50}$ mean value below or equal to $2000 \mathrm{mg} / \mathrm{kg}$ were identified as acutely toxic, whereas those with an acute oral $\mathrm{LD}_{50}$ mean value above $2000 \mathrm{mg} / \mathrm{kg}$ were identified as non-acutely toxic. Only the chemicals that fell into our group of toxic chemicals were considered in this second phase.

3. In a third phase, chemicals from the group of non-toxic chemicals that, nevertheless, had been assigned a harmonized classification (Annex VI of EU CLP Regulation) were identified and selected.

The mechanisms collected and shown in this report are not intended to be exhaustive.

Selection of chemicals with in vivo $L D_{50}$ values and in vitro cytotoxicity data

In the in-house database 178 test chemicals had oral $\mathrm{LD}_{50}$ values $^{10}$ that were collected from publicly available databases (e.g., ChemIDplus, IUCLID, RTECS, and HSDB), Merck index, EU Risk Assessment Reports, Sax's Dangerous Properties of Industrial Materials, and the published literature. According to the calculated mean LD $_{50}$ values, 112 test chemicals were assigned to an EU CLP acute oral toxicity category and 66 remain as non-toxic (i.e., no category assigned because the $\mathrm{LD}_{50}$ was higher than $2000 \mathrm{mg} / \mathrm{kg}$ ). Eleven out of the 66 non-classified chemicals had an official acute oral classification.

In vitro cytotoxicity data were available for 177 test chemicals that had been screened in the following international projects: NICEATM/ECVAM validation study (NIH, 2006), the EU FP6 project ACuteTox (Prieto et al., 2013a), and the ECVAM validation study (Prieto et al., 2013b). The list of chemicals used in each study is available through the JRC Chemical Lists of Information System (CheList $\left.{ }^{11}\right)$.

When the two sets were compared, in vitro cytotoxicity data were not available for two compounds, formaldehyde and carbon tetrachloride, and in vivo oral $\mathrm{LD}_{50}$ data were not found for benz(a)anthracene. Therefore, the final common set contained 176 chemicals.

\section{Results}

\subsection{Overall analysis of mechanistic maps}

The mechanistic information collected following the three-step strategy was visualized in maps according to the eight organs/ systems. The layout and structure across organs and systems

\footnotetext{
3 http://www.acutetox.eu/ (accessed 28.06.2018).

4 https://toxnet.nlm.nih.gov/newtoxnet/hsdb.htm (accessed 28.06.2018).

5 http://www.inchem.org/ (accessed 28.06.2018).

6 https://pubchem.ncbi.nlm.nih.gov/ (accessed 28.06.2018).

7 https://www.ncbi.nlm.nih.gov/pubmed (accessed 28.06.2018).

8 https://www.elsevier.com/solutions/scopus (accessed 28.06.2018).

9 https://scholar.google.it/ (accessed 28.06.2018).

10 Rat oral LD50 values (mg/kg body weight) collected for 178 chemicals in the context of the following international projects NICEATM/ECVAM validation study (NIH, 2006), the EU FP6 project ACuteTox (Hoffmann et al., 2010) and the ECVAM validation study (Prieto et al., 2013b): doi:10.14573/ altex.1805181s1

11 http://chelist.jrc.ec.europa.eu/ (accessed 28.06.2018).
} 
was harmonized and analyzed as shown below. Three maps were created per target organ/system. A first map illustrated the mechanisms found based on information collected from literature (step 1 under methods). The second map was an updated version based on the information collected from the in-house list of selected compounds (steps 2 and 3 under methods). The final harmonized version of each organ/system was shown by the third map (Fig. 1-8).

Information on mechanisms of toxicity was collected for 114 out of the 123 oral acutely toxic chemicals (see Methods). In terms of target organ/systems, the overall analysis summarized in Figure 9 shows that, according to the information found, the nervous and cardiovascular systems are the most frequent targets (67 and 39 chemicals, respectively) followed by liver, kidney, lung, gastrointestinal system, blood and immune system $(31,30$, $24,18,11$, and 3 chemicals, respectively). Twenty-six chemicals appear to target single organs, in particular the nervous systems (12 chemicals). Seventy-five chemicals affect more than one organ/system and thirteen chemicals affect all organs (non-specific target organ effects) (Fig. 10). Indirect effects were reported for 9 chemicals with multi-organ/system effects: 6 on the lung, 1 on the kidney, and 4 on the cardiovascular system (Fig. 9).

General cytotoxicity mechanisms were cited for 72 chemicals and target organ/system specific effects for 40 chemicals (11 chemicals acting on a single organ/system and 29 on multiple targets) (see Tab. 1). For pentachlorobenzene and tetramethylthiuram monosulfide, the specific mechanism of acute toxicity was not found.

Tables 2-9 provide an overview of the specific target organ/ system mechanisms leading to acute toxicity according to the information collected from the literature. The list of mechanisms shown is neither exhaustive nor definitive.

Referring to organ specific mechanisms of toxicity, interference with neurotransmitters and/or neurotransmission and impairment of propagation of electrical activity are among the main reported mechanisms for chemicals that target the nervous system. In particular, many chemicals interfere at the level of receptors and ion channel function.

Chemicals that target the cardiovascular system often interfere with ion balance/signaling/membrane potential of the cell and with intracellular signaling mechanisms.

For many of the chemicals that damage the liver after an acute insult, mechanisms such as depletion of free radical scavengers, ROS production, lipid peroxidation (grouped under oxidative stress induced inflammation), and necrosis were reported.

Alterations in kidney tubule cell structure (accumulation in proximal tubular cells, loss of tubular epithelial barrier, and/or tight junctions), alterations in tubule cell metabolism (interference with ion balance), tubular obstruction (impaired $\mathrm{Na}^{+}$and water reabsorption, distal cast formation, crystal deposition), and alterations in cell viability (necrosis) are among the most reported mechanisms leading to acute renal failure.

The in vivo classification for acute oral toxicity (i.e., the assigned CLP acute oral toxicity categories based on the collected mean oral $\mathrm{LD}_{50}$ values) of the chemicals acting via specific mechanisms of toxicity at organ/system level was evaluated in view of the information found in the literature for each chemical. Table 10 summarizes the outcome of this analysis, confirming that the nervous and cardiovascular systems are the most frequent targets for chemicals inducing acute oral toxicity.

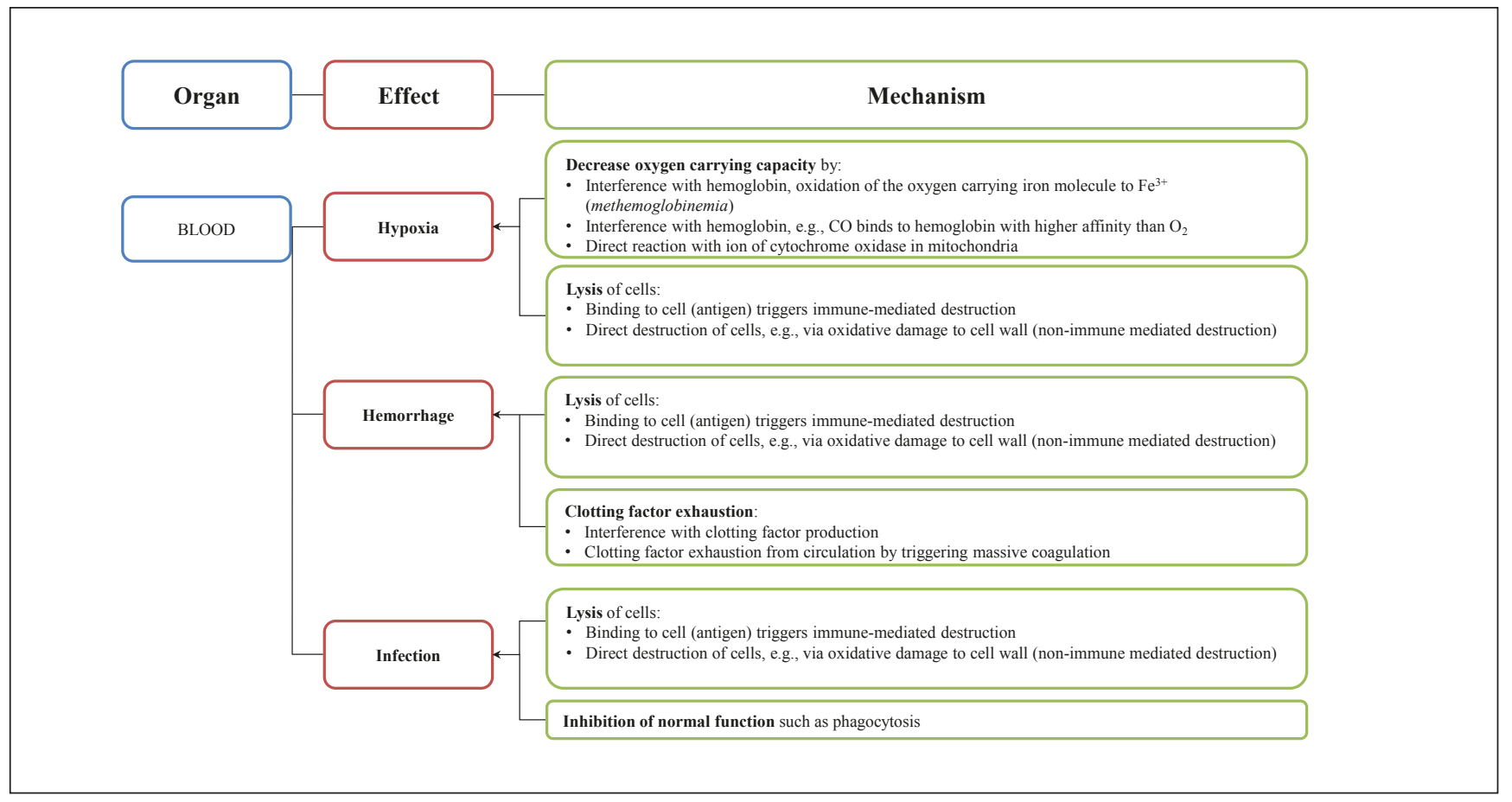

Fig. 1: Target organ blood - visualization of mechanisms leading to acute systemic toxicity $\mathrm{CO}$, carbon monoxide 


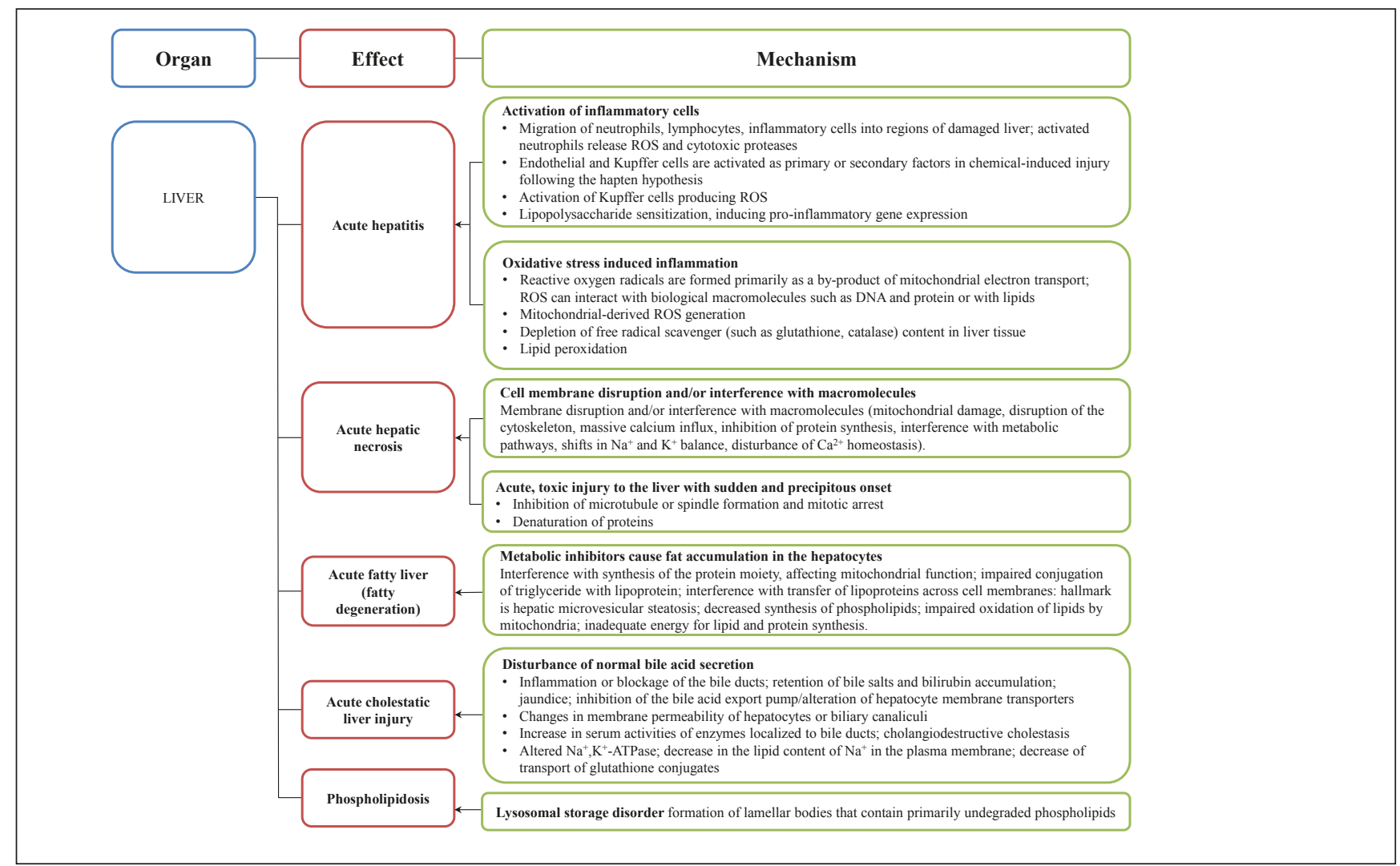

Fig. 2: Target organ liver - visualization of mechanisms leading to acute systemic toxicity

ROS, reactive oxygen species

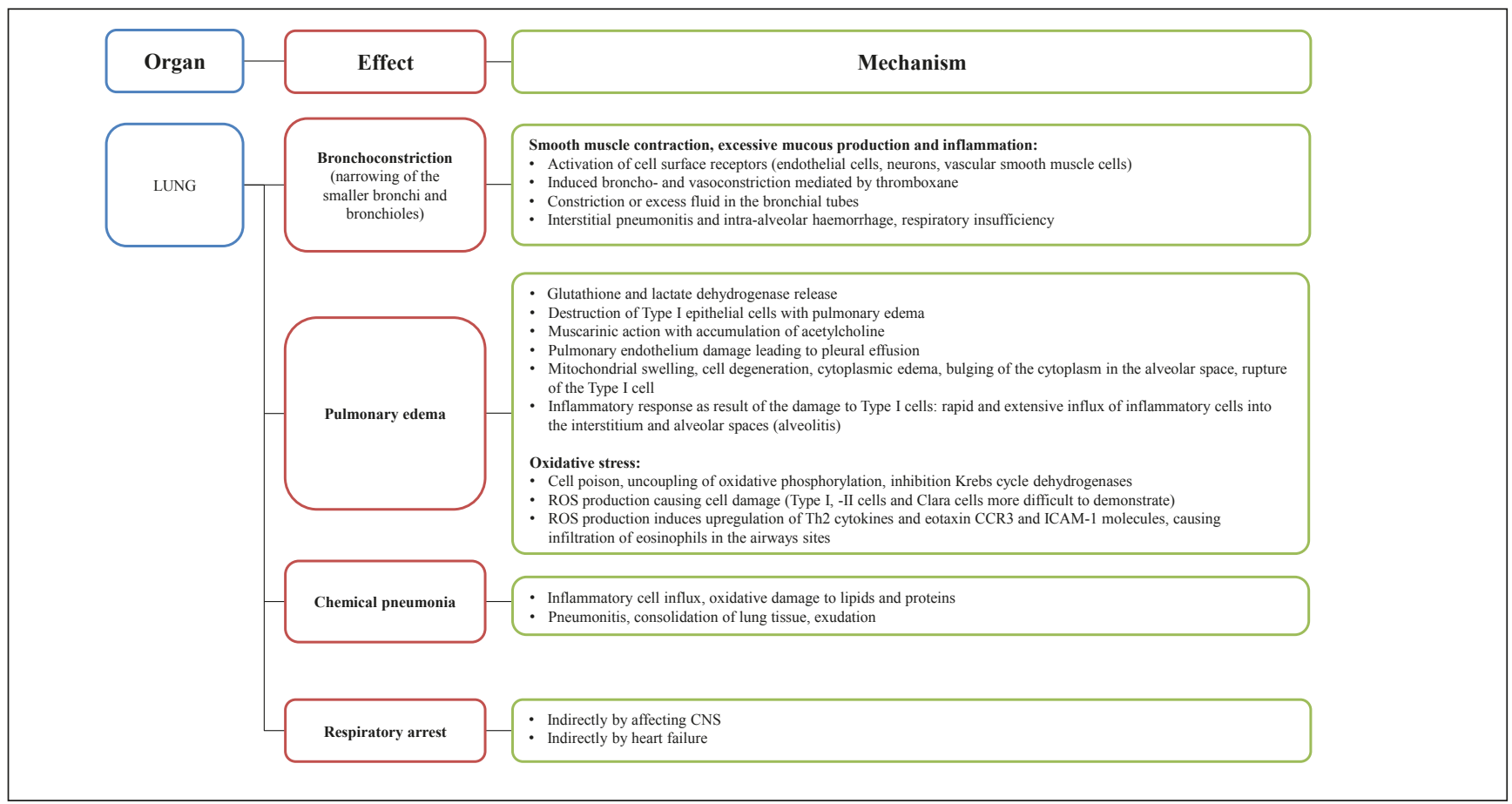

Fig. 3: Target organ lung - visualization of mechanisms leading to acute systemic toxicity

Th2, T helper type 2 cells; ROS, reactive oxygen species; CCR3, C-C motif chemokine receptor 3; ICAM-1, Intercellular Adhesion

Molecule 1; CNS, central nervous system 


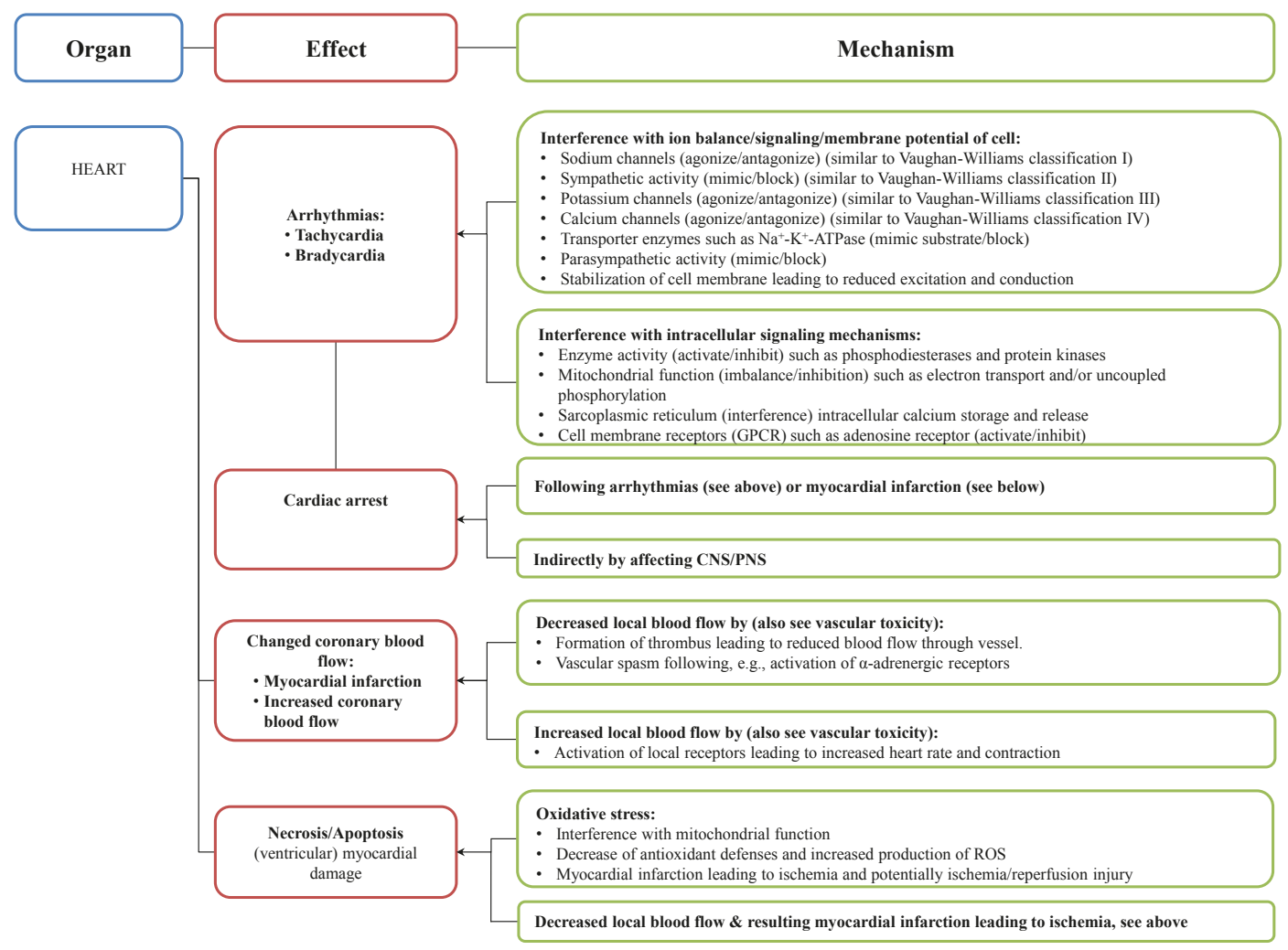

Organ Effect Mechanism

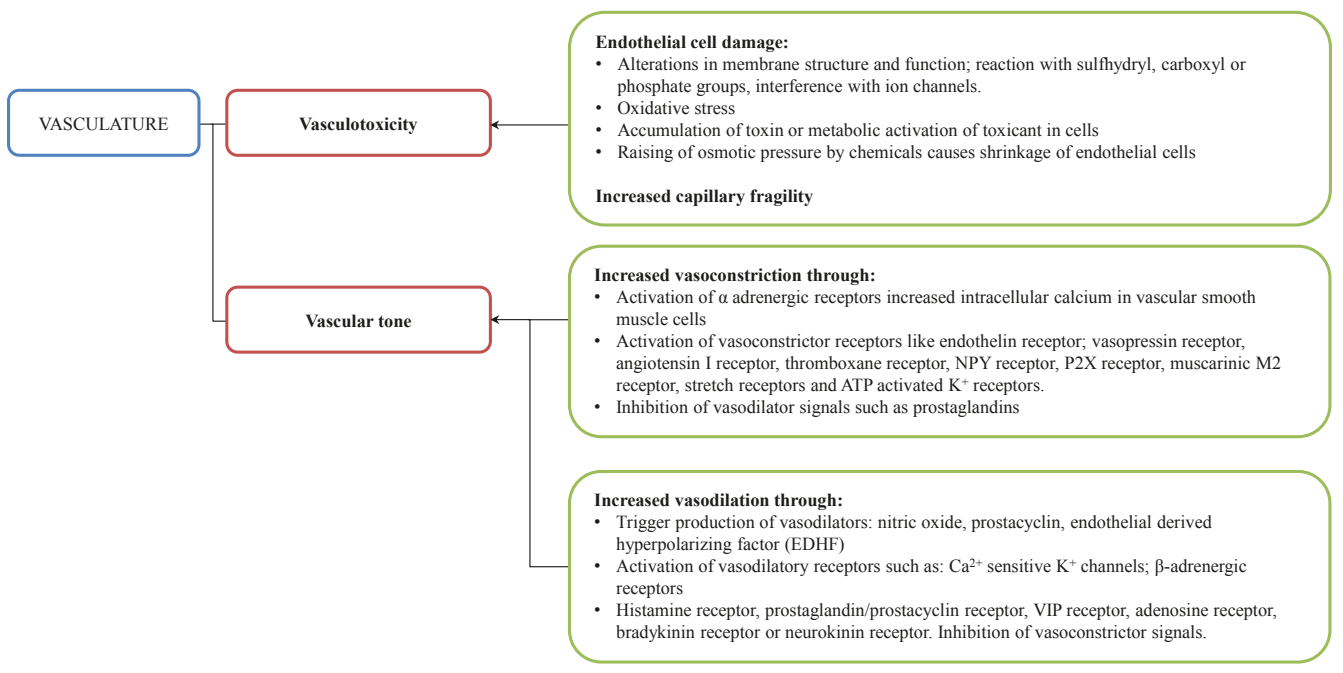

Fig. 4: Cardiovascular system - visualization of mechanisms leading to acute systemic toxicity GPCRs, G protein-coupled receptors; CNS, central nervous system; PNS, peripheral nervous system; ROS, reactive oxygen species; NPY, neuropeptide Y; P2X, purinergic receptors; M2, muscarinic acetylcholine receptor; VIP, vasoactive intestinal peptide 

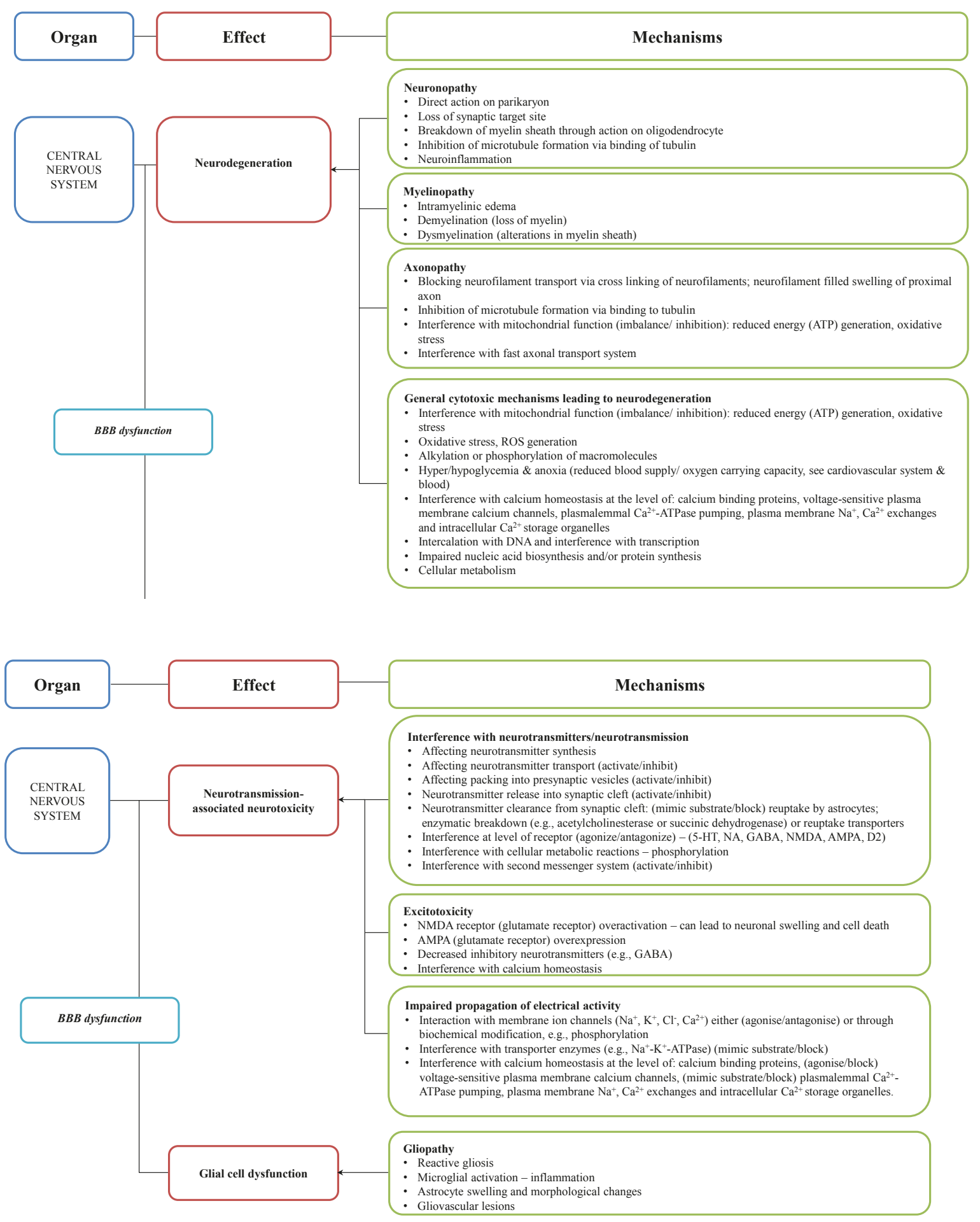


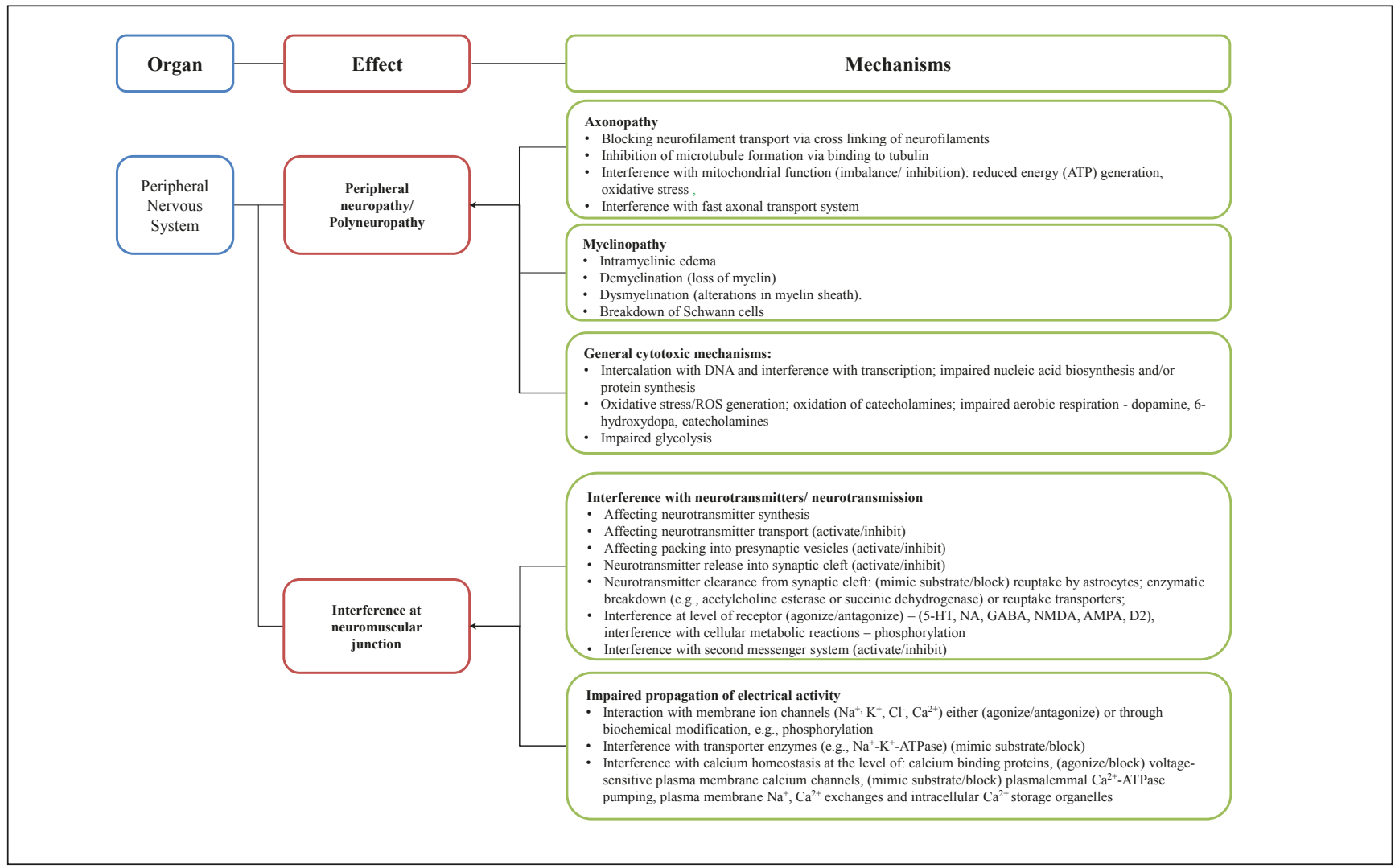

Fig. 5: Nervous system - visualization of mechanisms leading to acute systemic toxicity

ROS, reactive oxygen species; BBB, blood brain barrier; 5-HT, 5-hydroxytryptamine; NA, noradrenaline; GABA, gamma-aminobutyric acid; NMDA, N-methyl-D-aspartate; AMPA, a-amino-3-hydroxy-5-methylisoxazole-4-propionic acid; D2, dopamine

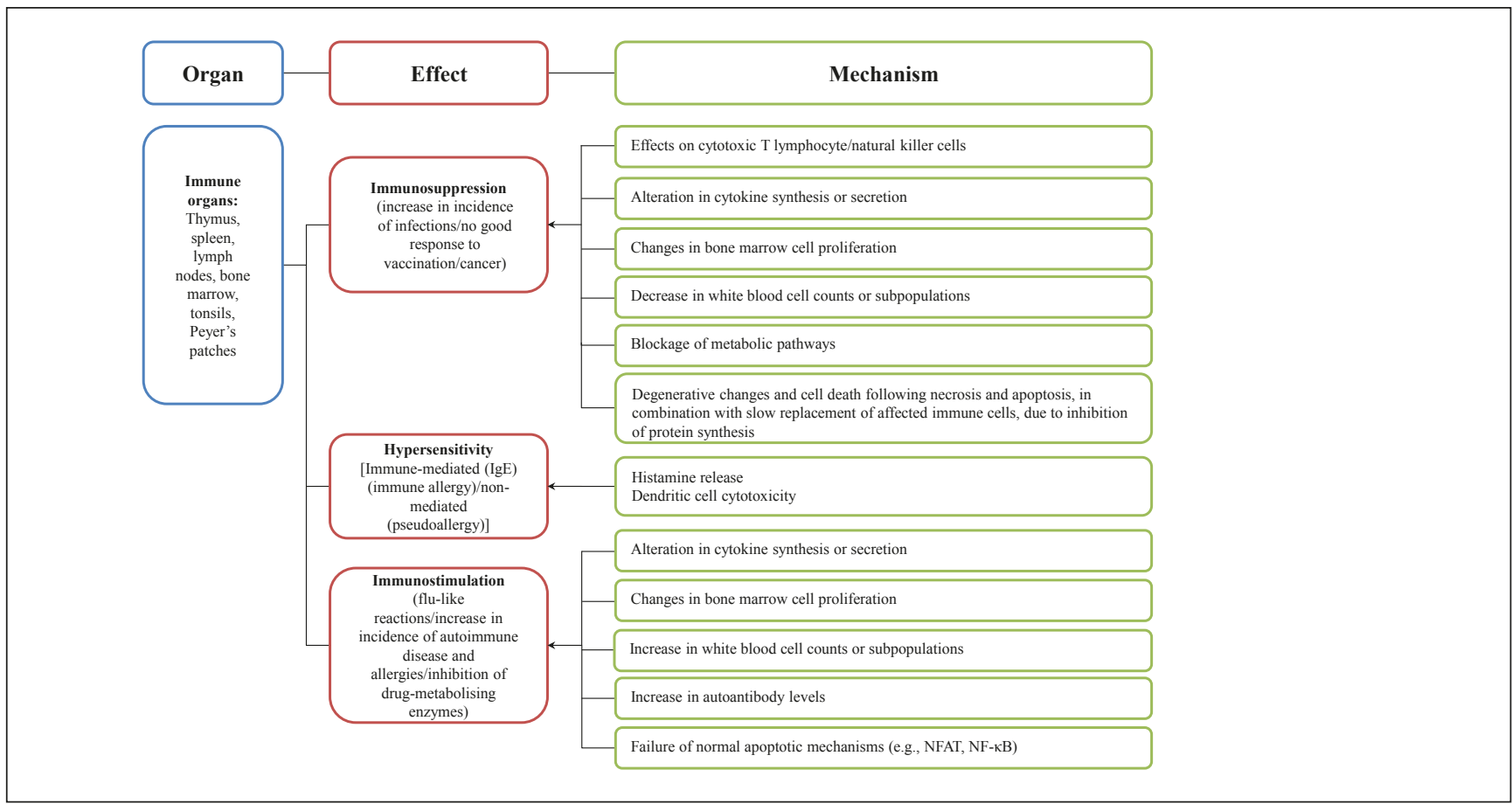

Fig. 6: Immune system - visualization of mechanisms leading to acute systemic toxicity

NFAT, nuclear factor of activated T-cells; NF-KB, nuclear factor kappa-light-chain-enhancer of activated B cells 


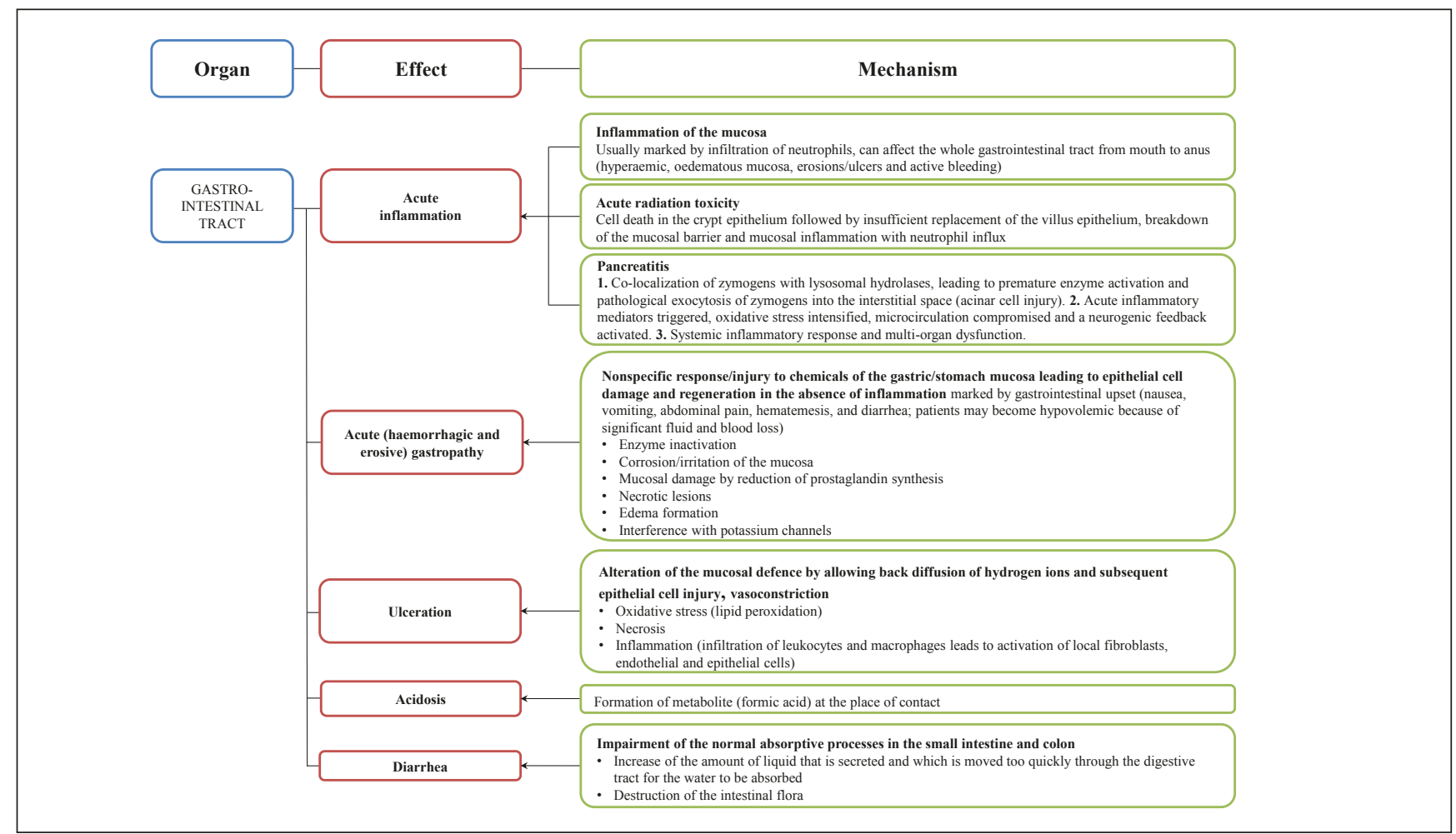

Fig. 7: Gastrointestinal system - visualization of mechanisms leading to acute systemic toxicity

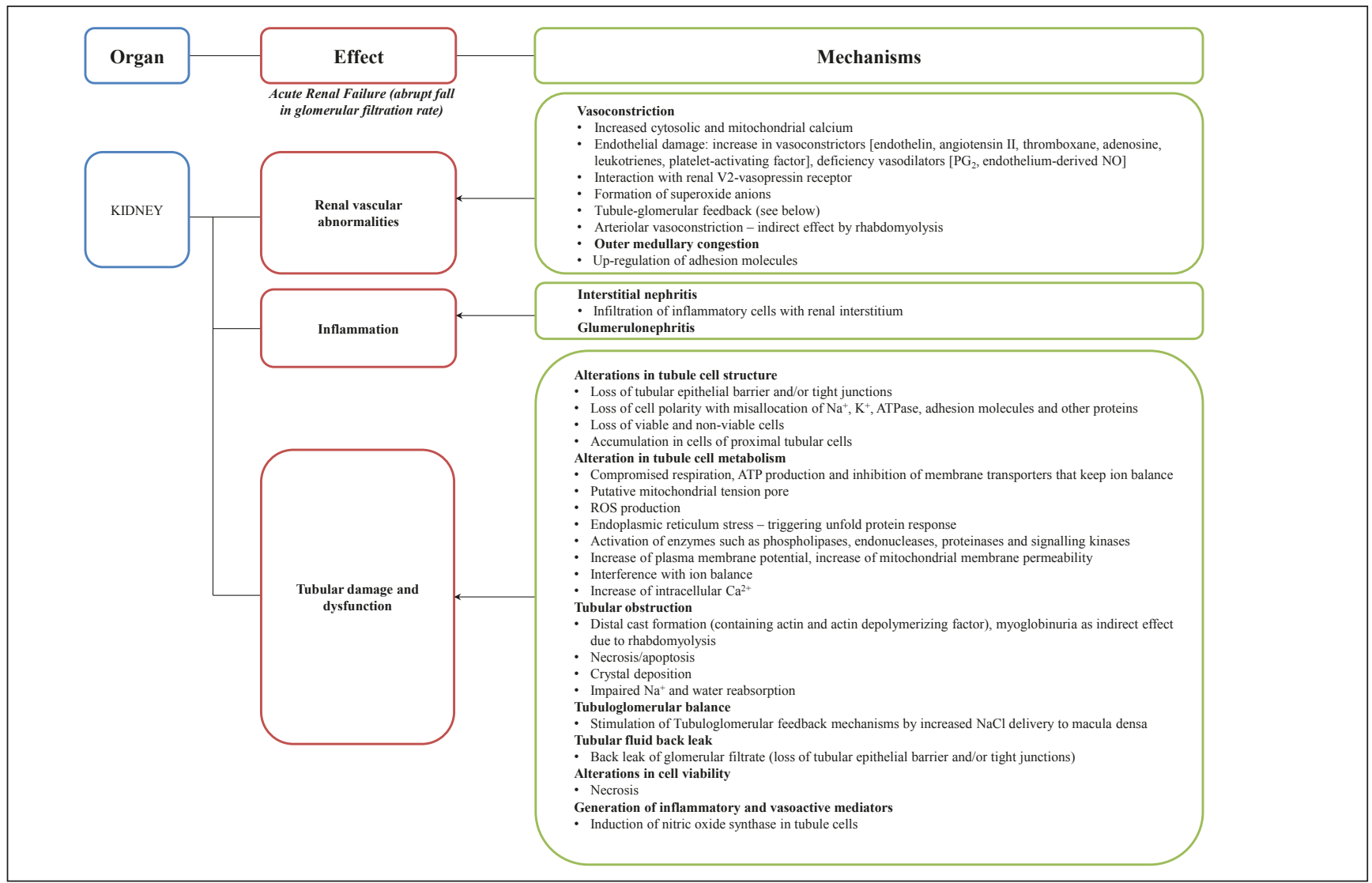

Fig. 8: Target organ kidney - visualization of mechanisms leading to acute systemic toxicity

ROS, reactive oxygen species 


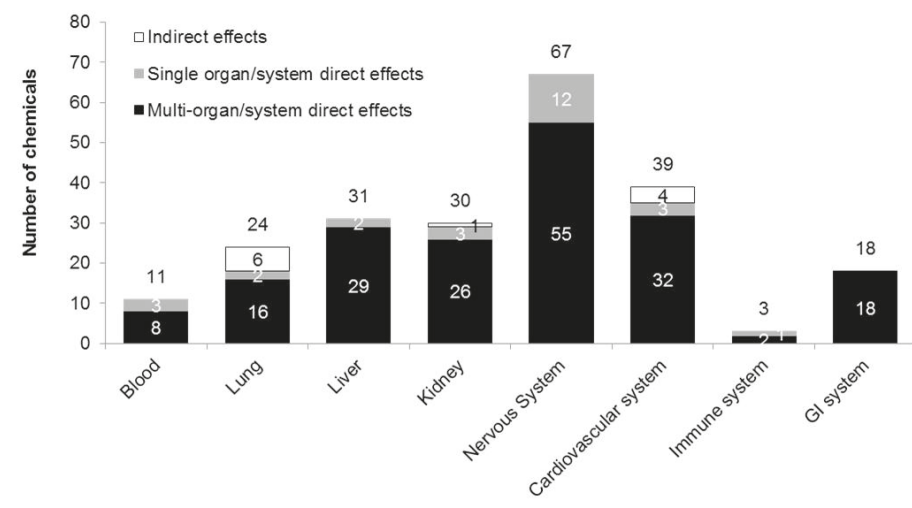

Fig. 9: Frequency of target organs/systems effects after acute oral insult

The number on top of each bar represents the number of chemicals affecting a particular organ/system. GI, gastrointestinal system

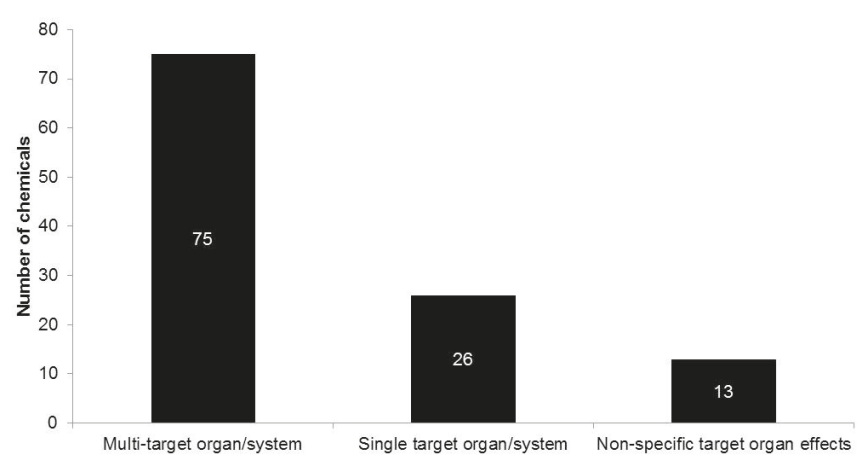

Fig. 10: Chemicals with specific target organs/systems effects (single and multiple) and non-specific effects Indirectly acting chemicals are included under multi-target organ/system. 
Tab. 1: Chemicals acting through general cytotoxic mechanisms and/or specific mechanisms of toxicity

\begin{tabular}{|c|c|c|}
\hline \multicolumn{2}{|l|}{ General cytotoxic mechanisms } & \multirow{2}{*}{$\begin{array}{l}\text { Specific mechanism of toxicity } \\
( \pm) \text {-Propranolol hydrochloride }\end{array}$} \\
\hline $\begin{array}{l}\text { (4-Ammonio-m-tolyl)ethyl } \\
\text { (2-hydroxyethyl) ammonium sulfate }\end{array}$ & Endosulfan & \\
\hline 1,2,3,4-Tetrachlorobenzene & Ethoxyquin & $( \pm)$-Verapamil hydrochloride \\
\hline 1,2,4-Trichlorobenzene & Ferrous sulfate & 1-Naphthylamine \\
\hline 1,2-Dichlorobenzene & Formaldehyde & 1-Phenyl-3-pyrazolidone \\
\hline 17a-Ethynyloestradiol & Glutethimide & 2,4,6-Tris(dimethylaminomethyl)phenol \\
\hline 1-Phenyl-2-thiourea & Haloperidol & 5,5-Diphenylhydantoin \\
\hline 2,4-Dichlorophenoxyacetic acid & Hexachlorophene & Acetophenone \\
\hline 4-Aminofolic acid & Isoniazid & Ammonium chloride \\
\hline 5-Fluorouracil & Lindane & Atropine sulfate monohydrate \\
\hline Acetaldehyde & Maleic acid & Codeine \\
\hline Acetylsalicylic acid & Malononitrile & D-Amphetamine \\
\hline Aconitine & Maprotiline & Diazepam \\
\hline Acrolein & Mercury II chloride & Diethylene glycol \\
\hline Acrylamide & Nicotine & Digoxin \\
\hline Amitriptilyne hydrochloride & Ochratoxin A & Diphenhydramine hydrochloride \\
\hline Arsenic trioxide & Octyl 3,4,5-trihydroxybenzoate & Disopyramide \\
\hline Barium chloride & Orphenadrine hydrochloride & Disulfoton \\
\hline Benzaldehyde & Paraquat dichloride & Epinephrine hydrogen tartrate \\
\hline Brucine & $\mathrm{p}$-Benzoquinone & Ethyl chloroacetate \\
\hline Busulfan & Pentachlorophenol & Ethylene glycol \\
\hline Cadmium (III) chloride & Phenanthrene & Fenpropathrin \\
\hline Caffeine & Phenol & Glufosinate-ammonium \\
\hline Carbon tetrachloride & Potassium cyanide & Lithium carbonate \\
\hline Chloral hydrate & Sodium arsenite & Lithium sulfate \\
\hline Chloroform & Sodium cyanate & Malathion \\
\hline Chloroquine bis(phosphate) & Sodium lauryl sulfate & Meprobamate \\
\hline Chlorpromazine & Sodium oxalate & Methadone hydrochloride \\
\hline cis-Diammineplatinum (II) dichloride & Sodium salt of chloroacetic acid & N-isopropyl-N'-phenyl-p-phenylenediamine \\
\hline Colchicine & Sodium selenate & Paraldehyde \\
\hline Copper sulfate & Sodium valproate & Parathion \\
\hline Cupric sulfate pentahydrate & Strychnine & Phenobarbital \\
\hline Cyclohexamide & Tert-butyl hydroperoxide & Physostigmine \\
\hline Cyclosporin A & Thallium sulfate & Procainamide hydrochloride \\
\hline Diallyl phthalate & Theophylline & Quinidine sulfate dehydrate \\
\hline Dichlorvos & Triethylenemelamine & Resorcinol \\
\hline Diquat dibromide & Valproic acid & Rifampicin \\
\hline & & Sodium pentobarbital \\
\hline & & Thioridazine hydrochloride \\
\hline & & Triphenyltin hydroxide \\
\hline & & Warfarin \\
\hline
\end{tabular}


Tab. 2: Specific mechanisms of acute blood toxicity

\begin{tabular}{|c|c|c|}
\hline Mechanisms & Example of chemicals & References \\
\hline \multicolumn{3}{|l|}{ Decrease oxygen carrying capacity } \\
\hline - Interference with hemoglobin & $\mathrm{N}$-isopropyl-N'-phenyl-p-phenylenediamine & Williamson et al., 1981 \\
\hline $\begin{array}{l}\text { - Oxidation of the oxygen carrying } \\
\text { iron molecule to } \mathrm{Fe}^{3^{+}}\end{array}$ & $\begin{array}{l}\text { Resorcinol } \\
\text { 1-Naphthylamine }\end{array}$ & $\begin{array}{l}\text { NJ RTK, } 2010 \\
\text { NJ RTK, } 2004\end{array}$ \\
\hline \multicolumn{3}{|l|}{ Clotting factor exhaustion } \\
\hline - Interference with clotting factor production & Warfarin & Hanley, 2004 \\
\hline \multicolumn{3}{|l|}{ Lysis of cells } \\
\hline $\begin{array}{l}\text { - Binding to cell (antigen) triggers } \\
\text { immune-mediated destruction }\end{array}$ & $\begin{array}{l}\text { Rifampicin } \\
\text { Quinidine sulfate }\end{array}$ & $\begin{array}{l}\text { POISINDEX }{ }^{\circledR} \text { System }{ }^{\text {a. }} \text {; Manika et al., } 2013 \\
\text { Freedman et al., } 1956\end{array}$ \\
\hline $\begin{array}{l}\text { - Direct destruction of cells, e.g., } \\
\text { via oxidative damage to cell wall }\end{array}$ & Copper sulfate & Franchitto et al., 2008 \\
\hline
\end{tabular}

a https://www.micromedexsolutions.com/home/dispatch (accessed 09.07.2018). Login required.

Tab. 3: Specific mechanisms of acute liver toxicity

\begin{tabular}{|l|l|l|}
\hline Mechanisms & Example of chemicals & References \\
\hline $\begin{array}{l}\text { Disturbance of normal bile acid } \\
\text { secretion }\end{array}$ & $17 \alpha$-Ethynyl estradiol & Wan and O'Brien, 2014; Davis et al., 1978 \\
\hline $\begin{array}{l}\text { - Changes in membrane permeability } \\
\text { of hepatocytes or biliary canaliculi }\end{array}$ & Chlorpromazine & Jennings et al., 2014 \\
\hline Fat accumulation in hepatocytes & Barium chloride & Ananda et al., 2013 \\
\hline $\begin{array}{l}\text { Membrane disruption and/or } \\
\text { interference with macromolecules }\end{array}$ & Isoniazid & $\begin{array}{l}\text { Saukkonen et al., 2006; } \\
\text { Boelsterli and Lee, 2014 }\end{array}$ \\
\hline $\begin{array}{l}\text { Inflammation induced by oxidative stress } \\
\text { • Lipid peroxidation }\end{array}$ & Carbon tetrachloride & El-Hadary and Ramadan Hassanien, 2016 \\
\hline
\end{tabular}

Tab. 4: Specific mechanisms of acute lung toxicity

\begin{tabular}{|l|l|l|}
\hline Mechanisms & Example of chemicals & References \\
\hline Pulmonary endothelium damage & 1-Phenyl-2-thiourea & Scott et al., 1990; Henderson et al., 2004 \\
\hline Increase capillary permeability & Dichlorvos & Li et al., 1989 \\
\hline Muscarinic action & Dichlorvos & Li et al., 1989 \\
\hline Destruction of Type I epithelial cells & Cadmium chloride & INCHEM, 2017 \\
\hline Inflammation & Chloroform & de Oliveira et al., 2015 \\
\hline $\begin{array}{l}\text { Induced broncho- and vasoconstriction } \\
\text { mediated by thromboxane }\end{array}$ & Tert-butyl hydroperoxide & Olafsdòttir et al., 1991 \\
\hline Intra-alveolar hemorrhage & Paraquat & Dinis-Oliveira, 2008 \\
\hline $\begin{array}{l}\text { Deposits of calcium oxalate crystals } \\
\text { in lung parenchyma }\end{array}$ & Ethylene glycol & Pomara et al., 2008; \\
\hline Irritation to respiratory tract & Acrolein & Bein and Leikauf, 2011 \\
\hline $\begin{array}{l}\text { Lung accumulation through } \\
\text { the polyamine uptake system }\end{array}$ & Paraquat & Dinis-Oliveira, 2008 \\
\hline
\end{tabular}


Tab. 5: Specific mechanisms of acute cardiovascular toxicity

\begin{tabular}{|c|c|c|}
\hline Mechanisms & Example of chemicals & References \\
\hline \multicolumn{3}{|c|}{ Interference with ion balance/signaling/membrane potential of cell } \\
\hline \multirow[t]{2}{*}{$\begin{array}{l}\text { - Mimic substrate/block transporter enzymes such as } \\
\mathrm{Na}^{+}-\mathrm{K}^{+} \text {-ATPase }\end{array}$} & Digoxin & $\begin{array}{l}\text { Nicolas et al., 2015; } \\
\text { Prassas et al., 2011 }\end{array}$ \\
\hline & Thallium sulphate & Riyaz et al., 2013 \\
\hline \multirow[t]{7}{*}{ - Interference with sodium and/or potassium channels } & Thallium sulphate & Riyaz et al., 2013 \\
\hline & Barium chloride & Bhoelan et al., 2014 \\
\hline & 5,5-Diphenylhydantoine & Ekwall et al., 1998 \\
\hline & Quinidine sulphate dehydrate & Kim and Benowitz, 1990 \\
\hline & Disopyramide & Kim and Benowitz, 1990 \\
\hline & Procainamide hydrochloride & Kim and Benowitz, 1990 \\
\hline & Amitriptyline hydrochloride & Woolf et al., 2007 \\
\hline - Interference with $\mathrm{Ca}^{2+}$ channels & Aconitine & Sun et al., 2014 \\
\hline \multirow[t]{2}{*}{ - QT interval prolongation } & Thioridazine hydrochloride & Beach et al., 2013 \\
\hline & Haloperidol & $\begin{array}{l}\text { Raudenska et al., 2013; } \\
\text { Henderson et al., } 1991\end{array}$ \\
\hline $\begin{array}{l}\text { - Calcium channel blocker and binding to the cytosolic } \\
\text { surface of the channel }\end{array}$ & Verapamil & $\begin{array}{l}\text { Nicolas et al., 2015; } \\
\text { Meister et al., } 2010\end{array}$ \\
\hline $\begin{array}{l}\text { - Stabilization of cell membrane leading to reduced } \\
\text { excitation and conduction }\end{array}$ & Chloroquine bis(phosphate) & Ekwall et al., 1998 \\
\hline - Mimic/block parasympathetic activity & Atropine sulphate & Ekwall et al., 1998 \\
\hline - Prevention of the reuptake of heart noradrenaline & Amitriptyline hydrochloride & Dollery, 1991; Ekwall et al., 1998 \\
\hline $\begin{array}{l}\text { - Pronounced negative chronotropic and inotropic effect and } \\
\text { a quinidine-like effect }\end{array}$ & Propranolol & Kerns et al., 1997 \\
\hline \multicolumn{3}{|l|}{ Interference with intracellular signaling mechanisms } \\
\hline - Interference with adenosine receptors & Caffeine & Ekwall et al., 1998 \\
\hline \multicolumn{3}{|l|}{ Increased vasoconstriction } \\
\hline $\begin{array}{l}\text { - Activation of } \beta 1 \text {-adrenergic receptors, } \beta 2 \text {-adrenergic } \\
\text { receptors in blood vessels }\end{array}$ & Epinephrine hydrogen tartrate & Zhang et al., 2011 \\
\hline Increased capillary fragility & Warfarin & Hanley, 2004 \\
\hline
\end{tabular}

Tab. 6: Specific mechanisms of acute neurotoxicity

\begin{tabular}{|c|c|c|}
\hline Mechanisms & Example of chemicals & References \\
\hline \multicolumn{3}{|l|}{ Interference with neurotransmitters/ neurotransmission } \\
\hline - Inhibition of glutamine synthetase and glutamate decarboxylase & Glufosinate ammonium & Lluís et al., 2008 \\
\hline - Inhibition of the dopamine transporter & Chloral hydrate & $\begin{array}{l}\text { Kreuter et al., 2004; } \\
\text { Sabeti et al., } 2003\end{array}$ \\
\hline $\begin{array}{l}\text { - Slowing down catecholamine metabolism by inhibiting } \\
\text { monoamine oxidase }\end{array}$ & D-amphetamine & Fitzgerald and Bronstein, 2013 \\
\hline \multicolumn{3}{|l|}{ Neurotransmitter release into synaptic cleft } \\
\hline $\begin{array}{l}\text { - Stimulation of glutamate release which can activate glutamate } \\
\text { receptors to initiate excitotoxic processes }\end{array}$ & Potassium cyanide & Patel et al., 1993 \\
\hline $\begin{array}{l}\text { - Stimulation of the release of norepinephrine and dopamine from } \\
\text { stores in adrenergic nerve terminals }\end{array}$ & D-amphetamine & Fitzgerald and Bronstein, 2013 \\
\hline $\begin{array}{l}\text { - Attenuation of glutamate release and reduction of activation } \\
\text { of glutamate receptors }\end{array}$ & Chloral hydrate & Kreuter et al., 2004 \\
\hline
\end{tabular}




\begin{tabular}{|c|c|c|}
\hline Mechanisms & Example of chemicals & References \\
\hline \multicolumn{3}{|l|}{ Neurotransmitter clearance from synaptic cleft } \\
\hline \multirow[t]{4}{*}{$\begin{array}{l}\text { - Inhibition of acetylcholinesterase and accumulation of } \\
\text { acetylcholine }\end{array}$} & Dichlorvos & $\begin{array}{l}\text { Binukumar and Gill, 2010; } \\
\text { EXETOXNET, 1999; } \\
\text { Sachana et al., } 2001\end{array}$ \\
\hline & Physostigmine & Gilman, 1985 \\
\hline & Disulfoton & ATSDR, 1995 \\
\hline & Parathion & Casarett and Doull, 2001 \\
\hline - Increased acetylcholine release at the neuromuscular junction & Phenol & Liao and Oehme, 1980 \\
\hline $\begin{array}{l}\text { - Blockage of the neuronal reuptake of norepinephrine, serotonin, } \\
\text { and dopamine }\end{array}$ & Amitriptilyne hydrochloride & Dollery, 1991; Ekwall et al., 1998 \\
\hline - Selective norepinephrine re-uptake blockade & Maprotiline & $\begin{array}{l}\text { Jan et al., 2013; } \\
\text { Baumann and Maître, } 1979\end{array}$ \\
\hline - Depletion of gamma-aminobutyric acid (GABA) & Isoniazid & Casarett and Doull, 2001 \\
\hline $\begin{array}{l}\text { - Increase of GABA by indirect mechanisms involving inhibition } \\
\text { of the enzyme succinate semialdehyde dehydrogenase (SSA-DH) } \\
\text { in the GABA shunt }\end{array}$ & Sodium valproate & $\begin{array}{l}\text { Sztajnkrycer, 2002; } \\
\text { Chateauvieux et al., } 2010\end{array}$ \\
\hline \multirow[t]{2}{*}{ Interference at level of receptor } & Phenobarbital & Jana et al., 2014 \\
\hline & Theophylline & Nakada et al., 1983 \\
\hline - Blockage of the action of acetylcholine at muscarinic receptors & Atropine sulfate monohydrate & Ekwall et al., 1998 \\
\hline - Competitive antagonism of cellular adenosine receptors & Caffeine & Fredholm et al., 1999 \\
\hline \multirow[t]{2}{*}{ - Antagonist at the glycine receptor } & Brucine & Teske et al., 2011 \\
\hline & Strychnine & Teske et al., 2011 \\
\hline $\begin{array}{l}\text { - Blocking the release of inhibitory neurotransmitters such } \\
\text { as GABA and acetylcholine }\end{array}$ & Codeine & $\begin{array}{l}\text { NClt, 2018; Takahama and } \\
\text { Shirasaki, } 2007\end{array}$ \\
\hline - Down-regulation of GABA receptors & Diazepam & Casarett and Doull, 2001 \\
\hline - Antagonizing chloride ion transport in GABA receptors & Endosulfan & Jang et al., 2016 \\
\hline - Interaction with GABAA receptors in a barbiturate-like fashion & Meprobamate & Rho et al., 1997 \\
\hline - Inhibition of NMDA receptors & Meprobamate & Rho et al., 1997 \\
\hline - Blockade of the GABA-receptor coupled sodium channel & Lindane & POISINDEX ${ }^{\circledR}$ System ${ }^{\mathrm{a}}$ \\
\hline - GABAA receptor agonist & Sodium pentobarbital & Dollery, 1991 \\
\hline - Inhibition of the reuptake of GABA into the glia and nerve endings & Valproic acid & $\begin{array}{l}\text { TOXNET, 2015 }{ }^{\text {b }} \text { POISINDEX } \\
\text { System }^{\text {a }}\end{array}$ \\
\hline \multirow[t]{3}{*}{ - Interference at the level of GABAA receptors } & Chloroform & $\begin{array}{l}\text { Dick, 2006; } \\
\text { Greenblatt and Meng, } 2001\end{array}$ \\
\hline & Phenobarbital & Jana et al., 2014 \\
\hline & Valproic acid & $\begin{array}{l}\text { Sztajnkrycer, 2002; } \\
\text { Chateauvieux et al., } 2010\end{array}$ \\
\hline - Anticholinergic effects & $\begin{array}{l}\text { Quinidine sulfate dehydrate } \\
\text { Disopyramide }\end{array}$ & Kim and Benowitz, 1990 \\
\hline - Competitive antagonism of acetylcholine at the neuroreceptor sites & Orphenadrine hydrochloride & $\begin{array}{l}\text { POISINDEX }{ }^{\circledR} \text { System }^{a} \text {; } \\
\text { Rejdak et al., } 2011\end{array}$ \\
\hline - Blockade of the H1-receptors & Diphenhydramine hydrochloride & Pragst et al., 2006 \\
\hline - Direct stimulation of $\alpha$ - and $\beta$-adrenergic receptors & D-amphetamine & Fitzgerald and Bronstein, 2013 \\
\hline - Glutamate receptor activation & Glufosinate ammonium & Matsumura et al., 2001 \\
\hline - Dopamine receptor antagonism & Chlorpromazine & Haddad and Winchester, 1990 \\
\hline - Blockage of dopamine D2 receptor & Thioridazine hydrochloride & POISINDEX ${ }^{\circledR}$ System ${ }^{a}$ \\
\hline - Competitive blockade of postsynaptic dopamine (D2) receptors & Haloperidol & Raudenska et al., 2013 \\
\hline
\end{tabular}




\begin{tabular}{|c|c|c|}
\hline Mechanisms & Example of chemicals & References \\
\hline $\begin{array}{l}\text { - NMDA antagonism and inhibition of serotonin/norepinephrine } \\
\text { reuptake }\end{array}$ & Methadone & $\begin{array}{l}\text { Zorn and Fudin, 2011; } \\
\text { Jamero et al., } 2011\end{array}$ \\
\hline - Agonist at nicotinic cholinergic receptors & Nicotine & Williams and Robinson, 1984 \\
\hline \multicolumn{3}{|l|}{ Impaired propagation of electrical activity } \\
\hline \multirow[t]{3}{*}{ - Interaction with membrane ion channels $\left(\mathrm{Na}^{+}, \mathrm{K}^{+}, \mathrm{Cl}^{-}, \mathrm{Ca}^{2+}\right)$} & 5,5-Diphenylhydantoin & Ekwall et al., 1998 \\
\hline & Aconitine & Chan, 2009; Peng et al., 2009 \\
\hline & Fenpropathrin & $\begin{array}{l}\text { Xiong et al., 2016; } \\
\text { Spencer et al., } 2001\end{array}$ \\
\hline \multirow[t]{2}{*}{$\begin{array}{l}\text { - Interference with transporter enzymes (e.g. } \mathrm{Na}^{+}-\mathrm{K}^{+} \text {-ATPase) } \\
\text { (mimic substrate/block) }\end{array}$} & Thallium sulfate & $\begin{array}{l}\text { Osorio-Rico et al., 2017; } \\
\text { Ekwall et al., 1998; } \\
\text { Casarett and Doull, } 2001\end{array}$ \\
\hline & Aconitine & Peng et al., 2009 \\
\hline $\begin{array}{l}\text { - Interference with the normal flux of } \mathrm{Na}^{+} \text {and } \mathrm{K}^{+} \text {ions across } \\
\text { the axon membrane as nerve impulses pass }\end{array}$ & Lindane & Vučević et al., 2009 \\
\hline \multicolumn{3}{|l|}{ Peripheral neuropathy/ Polyneuropathy } \\
\hline \multicolumn{3}{|l|}{ Myelinopathy } \\
\hline - Intramyelinic edema & Hexachlorophene & $\begin{array}{l}\text { Persson et al., 1978; } \\
\text { Casarett and Doull's, } 2001\end{array}$ \\
\hline \multicolumn{3}{|l|}{ Axonopathy } \\
\hline $\begin{array}{l}\text { - Blocking neurofilament transport via cross linking of } \\
\text { neurofilaments }\end{array}$ & Acrylamide & $\begin{array}{l}\text { Le Quesne, 1985; } \\
\text { LoPachin et al., } 2003\end{array}$ \\
\hline - Neurofilament filled swelling of proximal axon & Acrylamide & $\begin{array}{l}\text { Le Quesne, 1985; } \\
\text { LoPachin et al., } 2003\end{array}$ \\
\hline - Inhibition of microtubule formation via binding to tubulin & Colchicine & $\begin{array}{l}\text { Gooneratne et al., 2014; } \\
\text { Finkelstein et al., } 2010\end{array}$ \\
\hline
\end{tabular}

a http://www.thomsonhc.com

b https://toxnet.nlm.nih.gov/cgi-bin/sis/search/a?dbs+hsdb: @term+@ DOCNO+3582

\section{Tab. 7: Specific mechanisms of acute immune toxicity}

\begin{tabular}{|l|l|l|}
\hline Mechanisms & Example of chemicals & References \\
\hline $\begin{array}{l}\text { - Degenerative changes in combination with slow replacement } \\
\text { of affected immune cells due to inhibition of protein synthesis }\end{array}$ & Ochratoxin A & Al-Anati and Petzinger, 2006 \\
\hline - Decrease in whole blood cell counts or subpopulations & $\begin{array}{l}\text { Triethylene melamine } \\
\text { Triphenyl tin hydroxide }\end{array}$ & $\begin{array}{l}\text { Bickham et al., 1994 } \\
\text { Vos et al., 1984 }\end{array}$ \\
\hline - Changes in bone marrow cell proliferation & Triethylene melamine & Bickham et al., 1994 \\
\hline
\end{tabular}

Tab. 8: Specific mechanisms of acute gastrointestinal toxicity

\begin{tabular}{|l|l|l|}
\hline Mechanisms & Example of chemicals & References \\
\hline Epithelial cell damage & Colchicine & lacobuzio-Donahue et al., 2001 \\
\hline$\bullet$ Corrosion/irritation of the mucosa & Ferrous sulfate & Yuen and Gossman, 2018 \\
\hline - Interference with potassium channels & Barium chloride & Bhoelan et al., 2014 \\
\hline$\bullet$ Enzyme activation & Theophylline & Barnes, 2013 \\
\hline Inflammation of the mucosa & 5-Fluorouracil & Boussios et al., 2012 \\
\hline Formation of metabolite (formic acid) at the place of contact & Formaldehyde & $\begin{array}{l}\text { Wood, 2014; Pandey et al., 2000; } \\
\text { Eells et al., 1981 }\end{array}$ \\
\hline
\end{tabular}


Tab. 9: Specific mechanisms of acute kidney toxicity

\begin{tabular}{|c|c|c|}
\hline Mechanisms & Example of chemicals & References \\
\hline \multicolumn{3}{|l|}{ Vasoconstriction } \\
\hline - Arteriolar vasoconstriction indirect effect by rhabdomyolysis & $\begin{array}{l}\text { Codeine } \\
\text { Endosulfan } \\
\text { Brucine } \\
\text { Diphenhydramine }\end{array}$ & $\begin{array}{l}\text { Pokorny and Saunders, } 1994 \\
\text { Jang et al., } 2016 \\
\text { Teske et al., 2011; } \\
\text { Achappa et al., } 2012 \\
\text { Pragst et al., } 2006\end{array}$ \\
\hline - Deficiency vasodilators (PG2) & Acetylsalicylic acid & Ferenbach and Bonventre, 2016 \\
\hline - Endothelial damage with increase in vasoconstrictors & CsA & Bonventre, 2014 \\
\hline -Interaction with renal V2-vasopressin receptor & Lithium & Bonventre, 2003 \\
\hline Glumerulonephritis & Lithium & Naughton, 2008 \\
\hline Interstitial nephritis & Acetylsalicylic acid & Naughton, 2008 \\
\hline \multicolumn{3}{|l|}{ Alterations in tubule cell structure } \\
\hline - Accumulation in cells of proximal tubular cells & $\begin{array}{l}\text { Cisplatin } \\
\text { Cadmium chloride } \\
\text { Mercury chloride }\end{array}$ & $\begin{array}{l}\text { Bulacio and Torres, 2013; } \\
\text { Kuhlmann et al., } 1997 \\
\text { Ozbek, } 2012 \\
\text { Bonventre, 2003; Zhou et al., } 2008\end{array}$ \\
\hline - Loss of tubular epithelial barrier and/or tight junctions & Ochratoxin A & Gennari et al., 2004 \\
\hline \multicolumn{3}{|l|}{ Alterations in tubule cell metabolism } \\
\hline - Interference with ion balance & Ammonium chloride & McEvoy, 2006 \\
\hline \multicolumn{3}{|l|}{ Tubular obstruction } \\
\hline - Impaired $\mathrm{Na}^{+}$and water reabsorption & Cisplatin & Safirstein, 2004 \\
\hline - Distal cast formation & $\begin{array}{l}\text { Diethylene glycol } \\
\text { Ethylene glycol }\end{array}$ & $\begin{array}{l}\text { Fowles et al.,2017 } \\
\text { Fowles et al., 2017; Hess et al., } \\
\text { 2004; Huhn and Rosenberg, 1995; } \\
\text { Pomara et al., } 2008\end{array}$ \\
\hline - Crystal deposition and tubular obstruction & Sodium oxalate & Pawar and Vyawahare, 2017 \\
\hline Generation of inflammatory and vasoactive mediators & Cisplatin & Bonventre, 2003 \\
\hline \multicolumn{3}{|l|}{ Alterations in cell viability } \\
\hline - Necrosis of tubular epithelium & $\begin{array}{l}\text { Mercury chloride } \\
\text { 4-ammonio-m-tolyl)ethyl } \\
\text { (2-hydroxyethyl)ammonium } \\
\text { sulfate } \\
\text { Cadmium chloride } \\
\text { Brucine }\end{array}$ & $\begin{array}{l}\text { Zhou et al., } 2008 \\
\text { EPA TSCATS } \\
\text { Bonventre, } 2003 \\
\text { Liu et al., } 2015\end{array}$ \\
\hline
\end{tabular}

a https://bit.ly/2QkpbQ5

\subsection{Analysis of mechanistic information and in vitro 3T3 NRU cytotoxicity results}

A total of 97 chemicals, for which in vivo and in vitro cytotoxicity data were available, have been identified with target organ/ system specific effects, of which 91 were predicted as acutely toxic by the 3 T3 NRU cytotoxicity assay $\left(\mathrm{LD}_{50} \leq 2000 \mathrm{mg} / \mathrm{kg}\right)$. Table 11 summarizes the prediction of the acute oral toxicity (EU CLP toxicity categories) by the in vitro cytotoxicity assay for these chemicals.
Figure 11 complements Table 11 by adding the collected mechanistic information. It also shows the percentage of chemicals identified in vitro as acutely toxic acting through cell type specific mechanisms of toxicity and via general cytotoxic mechanisms when the acute oral toxicity category was correctly predicted (50 chemicals), under-predicted (32 chemicals), and over-predicted ( 9 chemicals) by the in vitro cytotoxicity assay.

An overview of the information collected with regard to specific target organ/system and general cytotoxicity for the chemicals 
Tab. 10: Contribution of specific target organ/system mechanisms of toxicity to the in vivo acute oral toxic category of chemicals

\begin{tabular}{|c|c|c|c|c|}
\hline $\begin{array}{l}\text { Organ specific mechanisms of } \\
\text { acute toxicity }\end{array}$ & $\begin{array}{l}{ }^{a} \text { CLP Cat. } 1 \\
\text { ( } 5 \text { chemicals) }\end{array}$ & $\begin{array}{l}{ }^{a} \text { CLP Cat. } 2 \\
\text { (15 chemicals) }\end{array}$ & $\begin{array}{l}{ }^{a} C L P \text { Cat. } 3 \\
\text { (26 chemicals) }\end{array}$ & $\begin{array}{l}\text { acLP Cat. } 4 \\
\text { (52 chemicals) }\end{array}$ \\
\hline Neurotoxicity & $3(60 \%)$ & $8(53 \%)$ & $13(50 \%)$ & $29(56 \%)$ \\
\hline Cardiovascular toxicity & 0 & $5(33 \%)$ & $5(19 \%)$ & $21(40 \%)$ \\
\hline Liver toxicity & 0 & $1(7 \%)$ & $4(15 \%)$ & $11(21 \%)$ \\
\hline Kidney toxicity & $1(20 \%)$ & $4(27 \%)$ & $4(15 \%)$ & $10(19 \%)$ \\
\hline Lung toxicity & $1(20 \%)$ & 0 & $7(27 \%)$ & $5(10 \%)$ \\
\hline Gastrointestinal toxicity & 0 & $2(13 \%)$ & $5(19 \%)$ & $4(8 \%)$ \\
\hline Blood toxicity & 0 & $2(13 \%)$ & 0 & $4(8 \%)$ \\
\hline Immune toxicity & $1(20 \%)$ & $1(7 \%)$ & $1(4 \%)$ & 0 \\
\hline
\end{tabular}

${ }^{a}$ Cat. $1: \leq 5 \mathrm{mg} / \mathrm{kg}$; Cat. 2: $>5 \mathrm{mg} / \mathrm{kg}, \leq 50 \mathrm{mg} / \mathrm{kg}$; Cat. $3:>50 \mathrm{mg} / \mathrm{kg}, \leq 300 \mathrm{mg} / \mathrm{kg}$; Cat. $4:>300 \mathrm{mg} / \mathrm{kg}, \leq 2000 \mathrm{mg} / \mathrm{kg}$

Tab. 11: Summary of prediction of EU CLP toxicity categories in vivo and in vitro for the set of chemicals classified for acute oral toxicity

Shadow cells indicate concordant predictions. EU CLP: EU regulation on classification, labelling and packaging of substances and mixtures; Cat: acute oral toxicity category; Cat. 1: rat oral $L_{50} \leq 5 \mathrm{mg} / \mathrm{kg}$; Cat. $2: 5 \mathrm{mg} / \mathrm{kg}<$ rat oral $L D_{50} \leq 50 \mathrm{mg} / \mathrm{kg}$; Cat. 3: $50 \mathrm{mg} / \mathrm{kg}<$ rat oral $\mathrm{LD}_{50} \leq 300 \mathrm{mg} / \mathrm{kg}$; Cat. 4: $300 \mathrm{mg} / \mathrm{kg}<$ rat oral $L_{50} \leq 2000 \mathrm{mg} / \mathrm{kg}$

\begin{tabular}{|l|l|l|l|l|}
\hline $\begin{array}{l}\text { 3T3 NRU predicted } \\
\text { toxicity (mg/kg) }\end{array}$ & \multicolumn{4}{|l|}{ Reference in vivo oral LD 50 (mg/kg) } \\
\hline & Cat. 1 & Cat. 2 & Cat. 3 & Cat. 4 \\
\hline Cat. 1 & 0 & 0 & 0 & 0 \\
\hline Cat. 2 & 1 & 1 & 1 & 0 \\
\hline Cat. 3 & 0 & 6 & 9 & 8 \\
\hline Cat. 4 & 4 & 6 & 15 & 40 \\
\hline
\end{tabular}

that are correctly assigned to the CLP acute oral toxicity category, under-predicted and over-predicted by the in vitro cytotoxicity assay, respectively, is provided in the supplementary information (Tab. S1, S2, and $\mathrm{S}^{12}$ ).

Among the 50 correctly predicted chemicals, 29 act through some general mechanisms of cytotoxicity $(58 \%)$ and 21 only via cell type specific mechanisms of toxicity (42\%).

Among the 32 under-predicted chemicals, 20 act through a general mechanism of cytotoxicity $(63 \%)$ and only 12 via cell type specific mechanisms of toxicity $(38 \%)$.

Among the 9 chemicals with toxicity category over-predicted by the cytotoxicity assay, 6 act through a general mechanism of cytotoxicity (67\%) and 3 only via specific mechanisms of toxicity $(33 \%)$.
Among the 6 chemicals falsely predicted as non-classified (Fig. $12)$, two act through some mechanism of general cytotoxicity $(33 \%)$ and four act only via cell type specific mechanisms of toxicity $(67 \%)$, as shown in the supplementary information (Tab. $\left.\mathrm{S} 4^{12}\right)$.

\subsubsection{Acute oral toxicity category 1}

From the compounds assigned in vivo to the acute oral toxicity category 1 (fatal if swallowed), three (i.e., brucine, disulfoton, and physostigmine) target the nervous system and act via specific mechanisms (e.g., inhibition of cholinesterase, antagonism of glycine receptor). Among the remaining compounds, 1-phenyl2-thiourea has the lung as target organ and needs bio-activation, and triethylenemelamine affects the immune system. General mechanisms of cytotoxicity have also been reported for these two compounds. For brucine, necrosis was found as the mechanism responsible for kidney tubular cell damage. When in vivo and in vitro mean values are compared, all compounds are misclassified by the in vitro cytotoxicity assay. Triethylene melamine is under-classified by one toxicity category and the other four compounds by 3 toxicity categories.

\subsubsection{Acute oral toxicity category 2}

Among the compounds assigned in vivo to the acute oral toxicity category 2 (fatal if swallowed), only colchicine is correctly predicted by the cytotoxicity assay. Colchicine inhibits microtubule formation and, thus, effectively inhibits mitosis, which is a general mechanism of toxicity. This mechanism of toxicity is also reported as the one responsible for the toxicity at the level of the nervous system and the liver. Digoxin and aconitine, which were predicted as false negatives by the cytotoxicity assay, act via specific mechanisms of toxicity such as interference with transporter enzymes (e.g., $\mathrm{Na}^{+}-\mathrm{K}^{+}$-ATPase) and calcium channels. Digoxin targets the cardiovascular system while aconitine

12 doi:10.14573/altex.1805181s2 


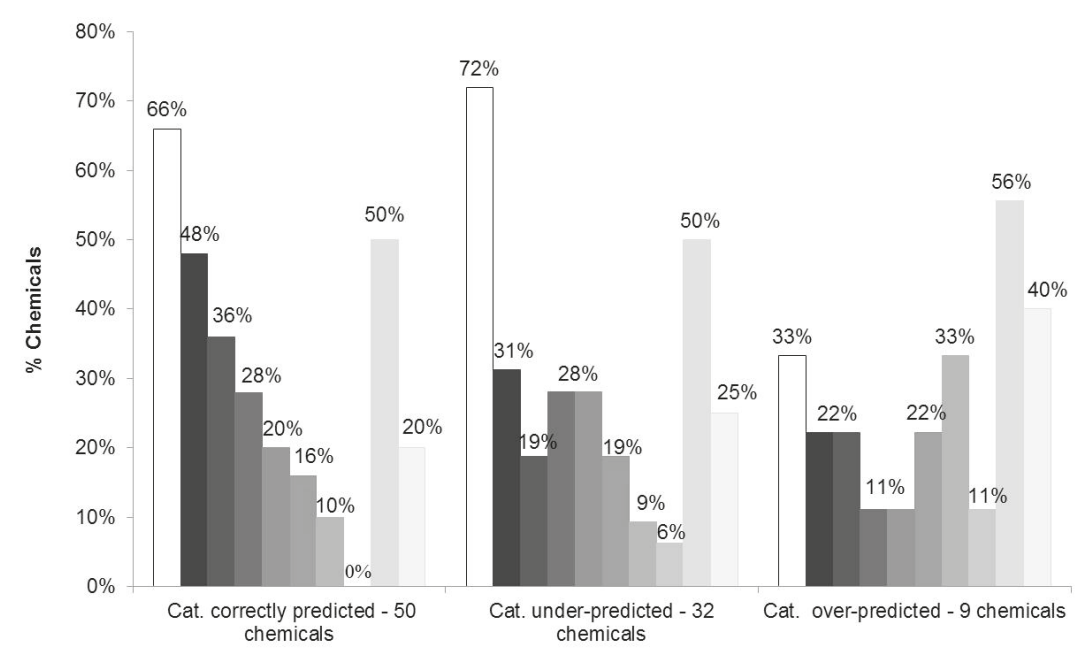

Fig. 11: Specific target organ/system toxicity and general cytotoxicity reported for the 91 chemicals predicted by the 3 T3 NRU cytotoxicity assay as positive chemicals (i.e., LD $_{50} \leq 2000 \mathrm{mg} / \mathrm{kg}$ )

Bars in each group represent from left to right: nervous system, cardiovascular system, liver, kidney, lung, gastrointestinal system, blood, immune system, general cytotoxicity, only specific target organ/systems. Cat., acute oral toxicity category

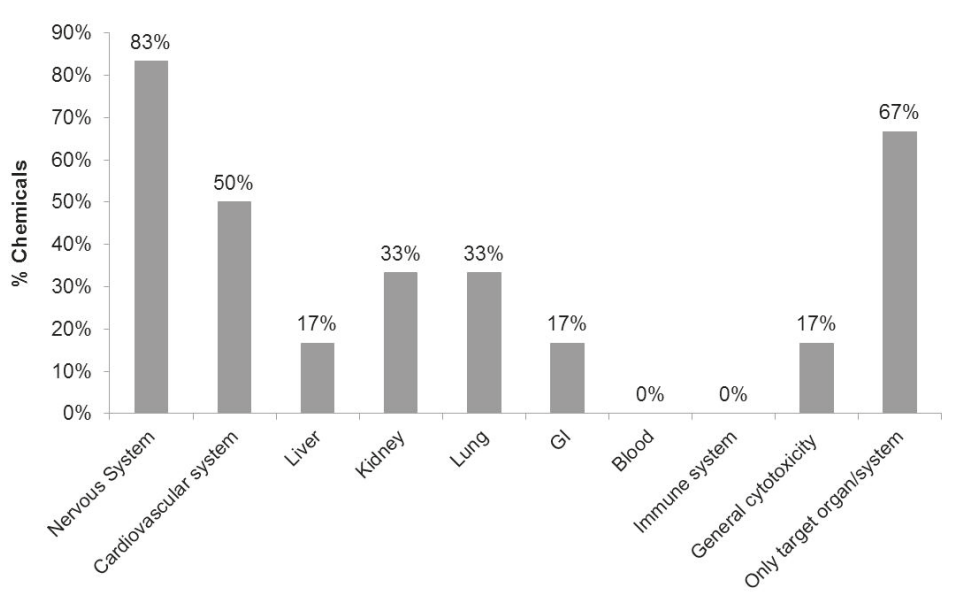

Fig. 12: Specific target organ/system toxicity and general cytotoxicity reported for the 6 chemicals falsely predicted as negatives by the 3T3 NRU cytotoxicity assay

$\mathrm{Gl}$, gastrointestinal system

acts on both the nervous and the cardiovascular system (Qiu et al., 2008; Chan, 2009; Prassas et al., 2011; Sun et al., 2014).

The compounds assigned to the toxicity category 2 in vivo but under-predicted in vitro act mainly via specific target organ toxicity mechanisms. For D-amphetamine, parathion, and strychnine, the central nervous system is the main target and specific mechanisms of toxicity were identified (i.e., inhibition of acetylcholinesterase, antagonism of glycine receptor, stimulation of the release of norepinephrine and dopamine). The kidney is the target for ochratoxin A, a well-known nephrotoxic agent acting on tubular cells (Ozbek, 2012). General cytotoxicity is also reported for ochratoxin $\mathrm{A}$, and necrosis has been found as the mechanism underlying strychnine effects on kidney tubular cell damage. Epinephrine hydrogen tartrate targets the cardiovascular system, while warfarin targets both the cardiovascular system and blood cells (Hanley, 2004; Klaassen, 2001).

\subsubsection{Acute oral toxicity category 3}

General mechanisms of toxicity were also described for the chemicals correctly assigned in vitro to the acute oral toxicity category 3 (toxic if swallowed). Only for ethyl chloroacetate no general mechanisms of toxicity were reported, although 
it is a moderate irritant via the oral route. For the compounds under-predicted in vitro by only one toxicity category, mechanisms of general cytotoxicity were reported. For sodium salt of chloroacetic acid and pentachlorophenol also the mechanisms identified at target organ level were general mechanisms of toxicity such as interference with mitochondrial function (CNS), membrane disruption and/or interference with macromolecules, depletion of free radical scavenger (such as glutathione, catalase) content in liver tissue, and compromised mitochondrial respiration of tubular cells (kidney). Dichlorvos and theophylline act through general mechanisms of cytotoxicity at CNS level, and also via specific mechanisms of toxicity. Seventy-three per cent of these under-predicted compounds are linked to the CNS as the target system (i.e., GABAA receptor agonist, blockade of the GABAA-receptor coupled sodium channel, interference with the normal flux of $\mathrm{Na}^{+}$and $\mathrm{K}^{+}$ions across the axon membrane during neuronal signaling, antagonism of N-methyl-D-aspartate (NMDA) receptors and inhibition of serotonin/norepinephrine reuptake, agonist of nicotinic cholinergic receptors, neurotransmitter clearance from synaptic cleft). Verapamil, barium chloride, and theophylline act at the level of the heart by different mechanisms (blocking the calcium channel and binding to the cytosolic surface of the channel; interference with potassium channels, interference with intracellular signaling mechanisms, such as enzymatic activity, e.g., phosphodiesterases and protein kinases). Fat accumulation in hepatocytes was also described as the liver specific mechanism of toxicity of barium chloride (Ananda et al., 2013).

\subsubsection{Acute oral toxicity category 4}

General mechanisms of toxicity are reported for $49 \%$ of the compounds correctly assigned to the acute oral toxicity category 4 (harmful if swallowed). For several of these compounds, general cytotoxicity was identified at the target organ/level alone or in addition to other specific mechanisms of toxicity (e.g., acetylsalicylic acid uncouples mitochondrial oxidative phosphorylation and also inhibits Krebs cycle dehydrogenases at CNS level (Ekwall et al., 1998); valproic acid alters the activity of the GABA neurotransmitter by increasing the inhibitory activity of GABA through inhibition of GABA degradation, inhibition of GABA transaminobutyrate, increased GABA synthesis, decreased turnover and inhibition of the GABA reuptake by the glia and synaptic mechanisms. It also interferes with cellular metabolic processes, interacts with membrane ion channels (Sztajnkrycer, 2002; Chateauvieux et al., 2010), and induces oxidative stress by compromising the antioxidant status of the neuronal tissue (Chaudhary and Parvez, 2012); caffeine in the CNS competitively antagonizes adenosine receptors, inhibits phosphodiesterase, stimulates catecholamine release, and increases free calcium and intracellular cAMP (Fredholm et al., 1999); orphenadrine chloride competitively antagonizes acetylcholine binding at the neuroreceptor sites and induces necrosis in liver (Sangster et al., 1978; Ekwall et al., 1998)). The nervous and the cardiovascular system appeared as targets for $70 \%$ and $54 \%$ of the harmful compounds, respectively. Among the compounds acting via specific mechanisms of toxicity, $65 \%(13 / 20)$ targeted both the nervous and the cardiovascular system.
Four harmful compounds were falsely predicted in vitro as non-acutely toxic. Of those, isoniazid and paraldehyde act via specific mechanisms such as interference at the level of CNS receptors and ion channel function, depletion of GABA (isoniazid), impairment of the propagation of electrical activity in the CNS (paraldehyde), and inflammation of the GI mucosa (paraldehyde) (Gilman, 1985; Carpentier et al., 1992). The harmful effects of ethylene glycol mainly result from the accumulation of its more toxic metabolites (Hess et al., 2004). Diethylene glycol is metabolized to 2-hydroxyethoxyacetaldehyde by alcohol dehydrogenase oxidation, then to 2-hydroxyacetic acid (HEAA) by aldehyde dehydrogenase. HEAA causes acidosis, renal failure, and neurologic dysfunction. It is thought that the parent compound is toxic as well (Schep et al., 2009). The formation of toxic metabolites will be missed in the in vitro cell system due to the lack of metabolic competence of the 3T3 cells. This could explain, at least in part, the misclassification by the in vitro approach.

Many of the harmful compounds are extensively or rapidly metabolized in the liver and toxic metabolites were reported for five compounds (quinidine sulfate dehydrate (Kim and Benowitz, 1990), chloroform (HSDB, ACuteTox project; Hodgson, 2004), chloral hydrate (Beland, 1999; Pershad et al., 1999; Dogan-Duyar et al., 2010), sodium valproate (ACuteTox project; Sztajnkrycer, 2002), and malathion (ACuteTox project; Simoneschi et al., 2014)).

\section{Discussion}

The exercise reported here served several purposes. First of all, the mechanistic information collected was visually summarized, allowing direct comparison across target organs/systems. Secondly, by organizing acute toxic effects by their mechanism and cell type(s), we could start to associate known acutely toxic compounds with the different mechanisms. Finally, organizing information in this manner should facilitate the development of AOPs and IATA, for example by identifying properties that are requisites of in vitro testing systems for specific target organ toxicity testing.

This work also aimed to identify specific (complementary) mechanisms of acute toxicity that are perhaps not covered by the validated 3T3 NRU cytotoxicity assay. Therefore, in our analysis we tried to address: (i) whether chemicals can be identified and classified based on either positive or negative specific effects on target organ(s) (according to the $2000 \mathrm{mg} / \mathrm{kg}$ threshold) using the 3T3 NRU assay; (ii) which organs are the most frequent targets; and (iii) whether the triggered pathways of toxicity are conserved across organs.

In the overall analysis, of the 97 chemicals identified with target organ specific effects, 94\% (91/97) were predicted as acutely toxic by the in vitro cytotoxicity assay and 6\% (6/97) as non-toxic. When comparing the positive (i.e., acutely toxic) and negative (i.e., non-acutely toxic) in vitro predictions with those of the in vivo study, it turned out that all six negatives were false predictions (false negatives), while $55 \%$ of the positive predictions were correctly predicted, according to a CLP acute toxicity category, 
$35 \%$ under-predicted and 10\% over-predicted. When evaluating the performance of any alternative approach for the purposes of regulatory classification, it is also necessary to consider the uncertainty associated with both the in vivo and the in vitro data. Actually, the analysis of consistency in classification published by Hoffmann et al. (2010) showed that conventional in vivo acute oral toxicity tests are intrinsically imprecise themselves and, about $44 \%$ of the substances would ambiguously occur within the limits of two adjacent classification categories (with at least $90 \%$ probability). A discussion of in vivo and in vitro data variability is outside the scope of this paper. Therefore, for the purpose of the mechanistic analysis shown and discussed here, the assignment of the compounds to the CLP acute oral toxicity categories was made based on the collected mean values (in vivo and in vitro). Another major source of uncertainty, not analyzed here due to lack of information, is the role of ADME (absorption, distribution, metabolism, and excretion) in determining the acute toxicity category. ADME has also been identified as a source of uncertainty in many OECD IATA case studies that are based on new approach methodologies. Several regulatory bodies have published guidance on the identification, characterization, and reporting of uncertainty (SCHEER, 2018).

A closer look at the chemicals acting through the specific target organs has not revealed a clear pattern with regard to which specific mechanisms of target organ toxicity are representative of compounds in the different CLP acute oral toxicity categories. For instance, approximately the same percentage of compounds acting through mechanisms of neurotoxicity was found in each acute oral toxicity category (i.e., $55 \%$ of the highly toxic chemicals allocated to toxicity categories 1 and 2, 50\% of the toxic chemicals in category 3 , and $56 \%$ of the harmful chemicals in category 4). A similar situation holds true for chemicals that act through mechanisms of cardiovascular toxicity, which were allocated to toxicity categories 2,3 , and $4(33 \%, 19 \%$, and $40 \%$, respectively). Mechanisms of nephrotoxicity were also found for chemicals in all toxicity categories. Mechanisms of liver, lung, and blood toxicity were described for a small percentage of the highly toxic compounds $(8 \%-21 \%)$. Based on these results, it can be concluded that mechanisms of toxicity specific for each organ can be triggered by compounds that belong to the different CLP acute oral toxicity categories. This is not surprising since the CLP categorization is based on potency, which can result from both toxicokinetic and toxicodynamic factors.

From the information collected and the analysis presented it can be concluded that general cytotoxicity is an important determinant of acute systemic toxicity. Overall, the majority of the analyzed chemicals $(63 \%)$ causing acute lethal toxicity act via some general (rather than organ specific) mechanisms of toxicity. The nervous and the cardiovascular systems are the most frequent targets, with changes in neurotransmission and altered ion flow being important mechanisms often associated with acute neurotoxicity and cardiotoxicity, respectively.

It is well recognized that the use of basal cytotoxicity alone to determine the acute toxicity of a chemical may not always be enough and, furthermore, it depends on the chemical's kinetic behavior and/or its specific mechanisms of toxicity. These features may need to be considered in order to correctly estimate the in vitro concentration causing toxicity that could be compared with the concentration that the target cells in vivo would be exposed to (EU FP6 project ACuteTox). In silico tools such as the Virtual Cell Based Assay (VCBA) can be used to simulate the distribution of the chemicals in the in vitro system (Zaldivar Comenges et al., 2017). By comparing the simulated and the nominal $\mathrm{IC}_{50}$ concentrations of the dissolved chemical, the influence of the in vitro kinetics on the cytotoxicity result may be anticipated. In addition, in vivo kinetics is an important determinant of acute systemic toxicity that requires further investigation (Graepel et al., 2017; Duarte Lopes Mascarenhas Proença et al., 2017). In silico tools such as Physiologically Based Kinetic (PBK) models (Paini et al., 2017) can be used to simulate the kinetics and distribution of chemicals in vivo.

Although specific target organ mechanisms of toxicity could in some cases explain the false negative prediction obtained with the cytotoxicity assay, in general it is difficult to explain in vitro misclassifications only on the basis of mechanistic information. Therefore, in addition to kinetic considerations, in vitro misclassifications could be also linked to the number of acute oral toxicity categories under CLP and the associated $\mathrm{LD}_{50}$ ranges, which are not based on a particular mechanistic rationale. Indeed, the outcome of the classification analysis carried out in the context of the EU FP6 project ACuteTox indicated that it is challenging to make a clear distinction between acute oral toxicity categories 1,2, and 3 based on in vitro concentration-response data (Kinsner-Ovaskainen et al., 2013) and, therefore, three levels of toxicity (i.e., level 1: combination of categories 1 to 3, level 2: category 4, and level 3: non-classified) were proposed (Prieto et al., 2013a). A similar grouping was also considered by Norlén et al. (2012) in an investigation of the predictive performances of five alternative approaches for the assessment of acute oral toxicity. Overall, the value of the CLP classification into four acute oral toxicity categories could be challenged.

Building on all the collected/generated information it would be worth trying to develop an alternative way of classifying chemicals for acute oral toxicity based mainly on cytotoxicity and kinetic information, and complemented, if needed, with relevant organ specific mechanisms of toxicity. Based on the mechanistic knowledge discussed in this paper, we propose to integrate in vitro assays anchored to the most frequent mechanisms of acute toxicity specific for each organ (CNS, heart, liver, and kidney) into an IATA. An IATA can include defined approaches, i.e., formalized decision-making approaches that apply fixed data interpretation procedures to data generated with a defined set of information sources (OECD, 2016b). In this regard, an in vitro cytotoxicity assay would be used together with specific target tissue toxicity mechanisms tested by assays permitting evaluation of neurotoxicity (as the most sensitive), followed by cardiotoxicity, hepatotoxicity, and kidney toxicity. Such a battery of tests should be designed to allow assessment of the compounds based on their cytotoxicity (e.g., based on the 3T3 NRU assay) and organ specific mechanisms. In the broader context of IATA, these in vitro mechanistic data should be integrated with additional sources of information (QSAR, read-across, in chemico, human data, in vivo data, etc.) including, where appropriate, exposure and ADME information. 
The development of AOPs relevant to acute neurotoxicity, cardiotoxicity, hepatotoxicity, etc., is already ongoing. However, as indicated, they are at different stages of development (Tab. S5 ${ }^{12}$ ). It is worth noting that some of the relevant AOPs are not specific to acute toxicity but nevertheless include key events that are relevant to acute exposure effects. Interestingly, some of the chemicals identified in these AOPs as triggers of molecular initiating events overlap with the chemicals reviewed in this report. The mechanistic information provided in this paper should inform the development of AOPs relevant to acute systemic toxicity, as well as AOP-informed IATA. The further development of AOPs and IATA should focus on the major target organs identified, i.e., the CNS, heart, liver, and kidney.

\section{References}

Achappa, B., Madi, D., Babu, Y. P. and Mahalingam, S. (2012). Rituals can kill - A fatal case of brucine poisoning. Australas Med J 5, 421-423. doi:10.4066/AMJ.2012.1293

Al-Anati, L. and Petzinger, E. (2006). Immunotoxic activity of ochratoxin A. J Vet Pharmacol Ther 29, 79-90. doi:10.1111/ j.1365-2885.2006.00718.x

Ananda, S., Shaohua, Z. and Liang, L. (2013). Fatal barium chloride poisoning: Four cases report and literature review. Am J Forensic Med Pathol 34, 115-118. doi:10.1097/PAF. 0b013e31828a2626

Andrew, D. J. (2013). Acute systemic toxicity: Oral, dermal and inhalation exposures In D. Allen and M. D. Waters (eds.), Reducing, Refining and Replacing the Use of Animals in Toxicity testing (183-214). Cambridge, UK: The Royal Society of Chemistry. doi:10.1039/9781849737920-00183

ATSDR - Agency for Toxic Substances and Disease Registry (1995). Toxicological Profile for Disulfoton. https://www.atsdr. cdc.gov/ToxProfiles/tp65.pdf

Barnes, P. J. (2013). Theophylline. Am J Respir Crit Care Med 188, 901-906. doi:10.1164/rccm.201302-0388PP

Baumann, P. A. and Maître, L. (1979). Neurobiochemical aspects of maprotiline (Ludiomil) action. J Int Med Res 7, 391400. doi:10.1177/030006057900700511

Beach, S. R., Celano, C. M., Noseworthy, P. A. et al. (2013). QTc prolongation, torsades de pointes, and psychotropic medications. Psychosomatics 54, 1-13. doi:10.1016/j.psym.2012. 11.001

Bein, K. and Leikauf, G. D. (2011). Acrolein - A pulmonary hazard. Mol Nutr Food Res 55, 1342-1360. doi:10.1002/mnfr. 201100279

Beland, F. A. (1999). Technical Report on the Toxicity and Metabolism Studies of Chloral Hydrate (CAS No. 302-17-0) Administered by Gavage to F344/N Rats and B6C3F Mice. National Toxicology Program Toxicity Report Series Number 59. https://ntp.niehs.nih.gov/ntp/htdocs/st_rpts/tox059.pdf

Bhoelan, B. S., Stevering, C. H., van der Boog, A. T. and van der Heyden, M. A. (2014). Barium toxicity and the role of the potassium inward rectifier current. Clin Toxicol (Phila) 52, 584-593. doi:10.3109/15563650.2014.923903

Bickham, J. W., Sawin, V. L., McBee, K. et al. (1994). Further flow cytometric studies of the effects of triethylenemelamine on somatic and testicular tissues of the rat. Cytometry 15, 222229. doi:10.1002/cyto. 990150307

Binukumar, B. K. and Gill, K. D. (2010). Cellular and molecular mechanisms of dichlorvos neurotoxicity: Cholinergic, nonchlolinergic, cell signaling, gene expression and therapeutic aspects. Indian J Exp Biol 48, 697-709.

Boelsterli, U. A. and Lee, K. K. (2014). Mechanisms of isoniazid-induced idiosyncratic liver injury: Emerging role of mitochondrial stress. J Gastroenterol Hepatol 29, 678-687. doi:10.1111/jgh.12516

Bonventre, J. V. (2003). Molecular response to cytotoxic injury: Role of inflammation, MAP kinases, and endoplasmic reticulum stress response. Semin Nephrol 23, 439-448. doi:10.1016/ S0270-9295(03)00115-3

Bonventre, J. V. (2014). Primary proximal tubule injury leads to epithelial cell cycle arrest, fibrosis, vascular rarefaction, and glomerulosclerosis. Kidney Int Suppl 4, 39-44. doi:10.1038/ kisup. 2014.8

Boussios, S., Pentheroudakis, G., Katsanos, K. and Pavlidis, N. (2012). Systemic treatment-induced gastrointestinal toxicity: Incidence, clinical presentation and management. Ann Gastroenterol 25, 106-118.

Buesen, R., Oberholz, U., Sauerm, U. G. and Landsiedel, R. (2016). Acute oral toxicity testing: Scientific evidence and practicability should govern Three Rs activities. Altern Lab Anim 44, 391-398.

Buesen, R., Oberholz, U., Sauer, U. G. and Landsiedel, R. (2018). Comment on "Alternative acute oral toxicity assessment under REACH based on sub-acute toxicity values". ALTEX 35, 119121. doi:10.14573/altex.1710111

Bulacio, R. P. and Torres, A. M. (2013). Organic anion transporter 5 (Oat5) renal expression and urinary excretion in rats treated with cisplatin: A potential biomarker of cisplatin-induced nephrotoxicity. Arch Toxicol 87, 1953-1962. doi:10.1007/ s00204-013-1062-0

Carpentier, P., Lallement, G., Bodjarian, N. et al. (1992). Effects of paraldehyde on the convulsions induced by administration of soman in rats. Fundam Clin Pharmacol 6, 309-318. doi:10.1111/j.1472-8206.1992.tb00125.x

Casarett, L. J. and Doull, J. (2001). Toxicology: The Basic Science of Poisons. New York, USA: McGraw-Hill Education Ltd.

Chan, T. Y. (2009). Aconite poisoning. Clin Toxicol (Phila) 47, 279-285. doi:10.1080/15563650902904407

Chapman, K., Creton, S., Kupferschmidt, H. et al. (2010). The value of acute toxicity studies to support the clinical management of overdose and poisoning: A cross-discipline consensus. Regul Toxicol Pharmacol 58, 354-359. doi:10.1016/j.yrtph. 2010.07.003

Chateauvieux, S., Morceau, F., Dicato, M. and Diederich, M. (2010). Molecular and therapeutic potential and toxicity of valproic acid. J Biomed Biotechnol 2010, 479364. doi:10. $1155 / 2010 / 479364$

Chaudhary, S. and Parvez, S. (2012). An in vitro approach to assess the neurotoxicity of valproic acid-induced oxidative stress in cerebellum and cerebral cortex of young rats. Neuroscience 225, 258-268. doi:10.1016/j.neuroscience.2012.08.060 
Clippinger, A. J., Allen, D., Jarabek, A. M. et al. (2018a). Alternative approaches for acute inhalation toxicity testing to address global regulatory and non-regulatory data requirements: An international workshop report. Toxicol In Vitro 48, 53-70. doi:10.1016/j.tiv.2017.12.011

Clippinger, A. J., Allen, D., Behrsing, H. et al. (2018b). Pathway-based predictive approaches for non-animal assessment of acute inhalation toxicity. Toxicol In Vitro 52, 131-145. doi:10. 1016/j.tiv.2018.06.009

Davis, R. A., Kern, F. Jr., Showalter, R. et al. (1978). Alterations of hepatic $\mathrm{Na}^{+}, \mathrm{K}^{+}$-ATPase and bile flow by estrogen: Effects on liver surface membrane lipid structure and function. Proc Natl Acad Sci U S A 75, 4130-4134. doi:10.1073/pnas. 75.9.4130

de Oliveira, T. H., Campos, K. K., Soares, N. P. et al. (2015). Influence of sexual dimorphism on pulmonary inflammatory response in adult mice exposed to chloroform. Int $J$ Toxicol 34, 250-257. doi:10.1177/1091581815580172

Dick, F. D. (2006). Solvent neurotoxicity. Occup Environ Med 63, 221-226. doi:10.1136/oem.2005.022400

Dinis-Oliveira, R. J., Duarte, J. A., Sánchez-Navarro, A. et al. (2008). Paraquat poisonings: Mechanisms of lung toxicity, clinical features, and treatment. Crit Rev Toxicol 38, 13-71. doi:10. 1080/10408440701669959

Dogan-Duyar, S., Willemse, J. L., Van Hee, P. et al. (2010). Chloral hydrate intoxication in a 3-month-old child: Avoidance of hemodialysis by an immediate determination of trichloroethanol. Clin Biochem 43, 328-330. doi:10.1016/j.clinbiochem. 2009.08.025

Dollery, S. C. (1991). Therapeutic Drugs. Philadelphia, USA: Churchill Livingstone.

Duarte Lopes Mascarenhas Proença, S., Paini, A., Joossens, E. et al. (2017). Application of the virtual cell based assay for simulation of in vitro chemical fate following acute esposure. EUR 28694. European Union, Luxembourg. http://publications.jrc. ec.europa.eu/repository/handle/JRC107407

ECHA - European Chemicals Agency (2017). Guidance on Information Requirements and Chemical Safety Assessment. Section R.7.4 of Chapter R.7a. Version 6.0. https://echa.europa.eu/ documents/10162/13632/information_requirements_r7a_ en.pdf

Eells, J. T., McMartin, K. E., Black, K. et al. (1981). Formaldehyde poisoning. Rapid metabolism to formic acid. JAMA 246, 1237-1238. doi:10.1001/jama.1981.03320110049029

El-Hadary, A. E. and Ramadan Hassanien, M. F. (2016). Hepatoprotective effect of cold-pressed Syzygium aromaticum oil against carbon tetrachloride (CCl4)-induced hepatotoxicity in rats. Pharm Biol 54, 1364-1372. doi:10.3109/13880209.2015. 1078381

Ekwall, B., Clemedson, C., Crafoord, B. et al. (1998). MEIC evaluation of acute systemic toxicity: Part V. Rodent and human toxicity data for the 50 reference chemicals. Altern Lab Anim 26, Suppl 2, 571-616.

Ekwall, B. (1999). Overview of the final MEIC results: II. The in vitro-in vivo evaluation, including the selection of a practical battery of cell tests for prediction of acute lethal blood concen- trations in humans. Toxicol In Vitro 13, 665-673. doi:10.1016/ S0887-2333(99)00061-2

EU - European Union (2006). Regulation (EC) No 1907/2006 of the European Parliament and the Council of 18 December 2006 concerning the Registration, Evaluation, Authorisation and Restriction of Chemicals (REACH), establishing a European Chemicals Agency, amending Directive 1999/45/EC and repealing Council Regulation (EEC) No 793/93 and Commission Regulation (EC) No 1488/94 as well as Council Directive 76/769/EEC and Commission Directives 91/155/EEC, 93/67/ EEC, 93/105/EC and 2000/21/EC. OJ L 396, 1-849. https:// eur-lex.europa.eu/legal-content/EN/TXT/PDF/?uri=CELEX: 02006R1907-20140410\& from $=\mathrm{EN}$

EU (2008). Regulation (EC) No 1272/2008 of the European Parliament and of the Council of 16 December 2008 on classification, labelling and packaging of substances and mixtures, amending and repealing Directives 67/548/EEC and 1999/45/ EC, and amending Regulation (EC) No 1907/2006. OJ L 353. http://eur-lex.europa.eu/legal-content/EN/TXT/PDF/?uri= CELEX:32008R1272\&from $=\mathrm{EN}$

EU (2009a). Regulation (EC) No 1223/2009 of the European Parliament and the Council of 30 November 2009 on cosmetic products. OJ L 342, 59-209. http://eur-lex.europa.eu/LexUri Serv/LexUriServ.do?uri=OJ:L:2009:342:0059:0209:en:PDF

EU (2009b). Regulation (EC) No 1107/2009 of the European Parliament and of the Council of 21 October 2009 concerning the placing of plant protection products on the market and repealing Council Directives 79/117/EEC and 91/414/EEC. OJ L 309, 1-47. https://eur-lex.europa.eu/legal-content/EN/TXT/ $\mathrm{PDF} /$ ?uri $=\mathrm{CELEX}: 32009 \mathrm{R} 1107 \&$ from $=\mathrm{EN}$

EU (2012). Regulation (EU) No 528/2012 of the European Parliament and of the Council of 22 May 2012 concerning the making available on the market and use of biocidal products. OJ L 167, 1-116. https://eur-lex.europa.eu/legal-content/EN/ TXT/PDF/?uri=CELEX:32012R0528\&from=EN

EURL ECVAM - European Union Reference Laboratory for alternatives to animal testing (2014). EURL ECVAM strategy to replace, reduce and refine the use of animals in the assessment of acute mammalian systemic toxicity. Report EUR 26704 EN. Luxembourg: Publications Office of the European Union. http:// publications.jrc.ec.europa.eu/repository/handle/JRC90611

EURL ECVAM (2015). EUR ECVAM Testing strategy for achieving 3Rs impact in the assessment of toxicokinetics and systemic toxicity, EUR 27315 EN. Luxembourg: Publications Office of the European Union. http://publications.jrc.ec.europa.eu/repository/handle/JRC96418

EXETOXNET - Extension Toxicology Network (1999). Pesticide Information Profile Diclorvos. http://extoxnet.orst.edu/ pips/dichlorv.htm.

Ferenbach, D. A. and Bonventre, J. V. (2016). Kidney tubules: Intertubular, vascular, and glomerular cross-talk. Curr Opin Nephrol Hypertens 25, 194-202. doi:10.1097/MNH. 0000000000000218

Finkelstein, Y., Aks, S. E., Hutson, J. R. et al. (2010). Colchicine poisoning: The dark side of an ancient drug. Clin Toxicol (Phila) 48, 407-414. doi:10.3109/15563650.2010.495348 
Fitzgerald, K. T. and Bronstein, A. C. (2013). Adderall ${ }^{\circledR}$ (amphetamine-dextroamphetamine) toxicity. Top Companion Anim Med 28, 2-7. doi:10.1053/j.tcam.2013.03.002

Fowles, J., Banton, M., Klapacz, J. and Shen, H. (2017). A toxicological review of the ethylene glycol series: Commonalities and differences in toxicity and modes of action. Toxicol Lett 278, 66-83. doi:10.1016/j.toxlet.2017.06.009

Franchitto, N., Gandia-Mailly, P., Georges, B. et al. (2008). Acute copper sulphate poisoning: A case report and literature review. Resuscitation 78, 92-96. doi:10.1016/j.resuscitation. 2008.02.017

Fredholm, B. B., Bättig, K., Holmén, J. et al. (1999). Actions of caffeine in the brain with special reference to factors that contribute to its widespread use. Pharmacol Rev 51, 83-133.

Freedman, A. L., Barr, P. S. and Brody, E. A. (1956). Hemolytic anemia due to quinidine: Observations on its mechanism. Am $J$ Med 20, 806-816. doi:10.1016/0002-9343(56)90164-4

Gennari, A., van den Berghe, C., Casati, S. et al. (2004). Strategies to replace in vivo acute systemic toxicity testing. The report and recommendations of ECVAM Workshop 50. ECVAM Workshop 50. Altern Lab Anim 32, 437-459.

Gilman, A. G. (1985). Goodman and Gilman's The Pharmacological Basis of Therapeutics. $7^{\text {th }}$ edition. USA Macmillan.

Gissi, A., Louekari, K., Hoffstadt, L. et al. (2017). Alternative acute oral toxicity assessment under REACH based on subacute toxicity values. ALTEX 34, 353-361. doi:10.14573/altex. 1609121

Gissi, A., Louekari, K., Hoffstadt, L. et al. (2018). Reply to comment on "Alternative acute oral toxicity assessment under REACH based on sub-acute toxicity values". ALTEX 35, 121122. doi:10.14573/altex.1712011

Gooneratne, I. K., Weeratunga, P., Caldera, M. and Gamage, R. (2014). Toxic encephalopathy due to colchicine - Gloriosa superba poisoning. Pract Neurol 14, 357-359. doi:10.1136/ practneurol-2013-000753

Graepel, R., Asturiol, D., Prieto, P. and Worth, A. P. (2016). Exploring waiving opportunities for mammalian acute systemic toxicity tests. Altern Lab Anim 44, 271-279.

Graepel, R., Lamon, L. and Asturiol, D. (2017). The virtual cell based assay: Current status and future perspectives. Toxicol In Vitro 45, 258-267. doi:10.1016/j.tiv.2017.01.009

Greenblatt, E. P. and Meng, X. (2001). Divergence of volatile anesthetic effects in inhibitory neurotransmitter receptors. Anesthesiology 94, 1026-1033. doi:10.1097/00000542200106000-00017

Haddad, L. M. and Winchester, J. F. (1990). Clinical Management of Poisoning and Drug Overdose. Philadelphia, USA.

Halle, W. (2003). The registry of cytotoxicity: Toxicity testing in cell cultures to predict acute toxicity (LD50) and to reduce testing in animals. Altern Lab Anim 31, 89-198.

Hamm, J., Sullivan, K. and Clippinger, A. J. (2017). Alternative approaches for identifying acute systemic toxicity: Moving from research to regulatory testing. Toxicol In Vitro 41, 245259. doi:10.1016/j.tiv.2017.01.004

Hanley, J. P. (2004). Warfarin reversal. J Clin Pathol 57, 11321139. doi:10.1136/jcp.2003.008904
Henderson, R. A., Lane, S. and Henry, J. A. (1991). Life-threatening ventricular arrhythmia (torsades de pointes) after haloperidol overdose. Hum Exp Toxicol 10, 59-62. doi:10. 1177/096032719101000110

Henderson, M. C., Krueger, S. K., Stevens, J. F. and Williams, D. E. (2004). Human flavin-containing monooxygenase form 2 S-oxygenation: Sulfenic acid formation from thioureas and oxidation of glutathione. Chem Res Toxicol 17, 633-640. doi:10.1021/tx034253s

Hess, R., Bartels, M. J. and Pottenger, L. H. (2004). Ethylene glycol: An estimate of tolerable levels of exposure based on a review of animal and human data. Arch Toxicol 78, 671-680. doi:10.1007/s00204-004-0594-8

Hodgson, E. (2004). A Textbook of Modern Toxicology. New Jersey, USA: Wiley. doi:10.1002/0471646776

Hoffmann, S., Kinsner-Ovaskainen, A., Prieto, P. et al. (2010). Acute oral toxicity: Variability, reliability, relevance and interspecies comparison of rodent LD50 data from literature surveyed for the ACuteTox project. Regul Toxicol Pharmacol 58, 395-407. doi:10.1016/j.yrtph.2010.08.004

Huhn, K. M. and Rosenberg, F. M. (1995). Critical clue to ethylene glycol poisoning. CMAJ 152, 193-195.

Iacobuzio-Donahue, C. A., Lee, E. L., Abraham, S. C. et al. (2001). Colchicine toxicity: Distinct morphologic findings in gastrointestinal biopsies. Am J Surg Pathol 25, 1067-1073. doi:10.1097/00000478-200108000-00012

ICH - International Conference on Harmonisation (2000). Safety pharmacology studies for human pharmaceuticals S7A. http:// www.ich.org/products/guidelines/safety/safety-single/article/ safety-pharmacology-studies-for-human-pharmaceuticals.html ICH (2009). Guidance on Nonclinical Safety Studies for the Conduct of Human Clinical Trials and Marketing Authorization for Pharmaceuticals M3(R2). http://www.ich.org/products/ guidelines/safety/safety-single/article/guidance-on-nonclinical-safety-studies-for-the-conduct-of-human-clinical-trialsand-marketing-author.html

INCHEM - International Programme on Chemical Safety (2017). Cadmium. Prepared by an international group of experts on behalf of ILO and WHO, with the financial assistance of the European Commission. http://www.inchem.org/documents/ icsc/icsc/eics0116.htm.

Jamero, D., Borghol, A., Vo, N. and Hawawini, F. (2011). The emerging role of NMDA antagonists in pain management. US Pharm 36, HS4-HS8. https://www.uspharmacist.com/article/ the-emerging-role-of-nmda-antagonists-in-pain-management

Jan, C. R., Su, J. A., Teng, C. C. et al. (2013). Mechanism of maprotiline-induced apoptosis: Role of $\left[\mathrm{Ca}^{2+}\right](\mathrm{i}), \mathrm{ERK}, \mathrm{JNK}$ and caspase-3 signaling pathways. Toxicology 304, 1-12. doi:10.1016/j.tox.2012.11.013

Jana, S., Chakravarty, C. Taraphder, A. and Ramasubban, S. (2014). Successful use of sustained low efficiency dialysis in a case of severe phenobarbital poisoning. Indian J Crit Care Med 18, 530-532. doi:10.4103/0972-5229.138159

Jang, T., Jang, J. and Lee, K. (2016). Mechanism of acute endosulfan intoxication-induced neurotoxicity in Sprague-Dawley rats / Mehanizam akutne neurotoksičnosti u Sprague-Dawley 
štakora izazvane trovanjem endosulfanom. Archives of Industrial Hygiene and Toxicology 67, 9-17. doi:10.1515/aiht-201667-2702

Jennings, P., Schwarz, M., Landesmann, B. et al. (2014). SEURAT-1 liver gold reference compounds: A mechanism-based review. Arch Toxicol 88, 2099-2133. doi:10.1007/s00204-0141410-8

Kerns, W., Ransom, M., Tomaszewski, C. and Raymond, R. (1997). The effect of hypertonic sodium and dantrolene on propranolol cardiotoxicity. Acad Emerg Med 4, 545-551. doi:10.1111/j.1553-2712.1997.tb03576.x

Klaassen, C. (2001). Casarett and Doull's Toxicology: The Basic Science of Poisons. New York, USA: McGraw-Hill.

Kim, S. Y. and Benowitz, N. L. (1990). Poisoning due to class IA antiarrhythmic drugs: Quinidine, procainamide and disopyramide. Drug Saf 5, 393-420. doi:10.2165/00002018199005060-00002

Kinsner-Ovaskainen, A., Prieto, P., Stanzel, S. and Kopp-Schneider, A. (2013). Selection of test methods to be included in a testing strategy to predict acute oral toxicity: An approach based on statistical analysis of data collected in phase 1 of the ACuteTox project. Toxicol In Vitro 27, 1377-1394. doi:10. 1016/j.tiv.2012.11.010

Kuhlmann, M. K., Burkhardt, G. and Köhler, H. (1997). Insights into potential cellular mechanisms of cisplatin nephrotoxicity and their clinical application. Nephrol Dial Transplant 12, 2478-2480. doi:10.1093/ndt/12.12.2478

Kreuter, J. D., Mattson, B. J., Wang, B. et al. (2004). Cocaine-induced Fos expression in rat striatum is blocked by chloral hydrate or urethane. Neuroscience 127, 233-242. doi:10.1016/j. neuroscience.2004.04.047

Le Quesne, P. M. (1985). Clinical and morphological findings in acrylamide toxicity. Neurotoxicology 6, 17-24.

Leth, P. M. and Gregersen, M. (2005). Ethylene glycol poisoning. Forensic Sci Int 155, 179-184. doi:10.1016/j.forsciint.2004.11.012

Li, C., Miller, W. T. and Jiang, J. (1989). Pulmonary edema due to ingestion of organophosphate insecticide. AJR Am J Roentgenol 152, 265-266. doi:10.2214/ajr.152.2.265

Liao, J. T. and Oehme, F. W. (1980). Literature reviews of phenolic compounds. I. Phenol. Vet Hum Toxicol 22, 160-164.

Liu, F., Wang, X., Han, X. et al. (2015). Cytotoxicity and DNA interaction of brucine and strychnine - Two alkaloids of semen strychni. Int J Biol Macromol 77, 92-98. doi:10.1016/j. ijbiomac.2015.03.017

LoPachin, R. M., Balaban, C. D. and Ross, J. F. (2003). Acrylamide axonopathy revisited. Toxicol Appl Pharmacol 188, 135-153. doi:10.1016/S0041-008X(02)00072-8

Lluís, M., Nogué, S. and Miró, O. (2008). Severe acute poisoning due to a glufosinate containing preparation without mitochondrial involvement. Hum Exp Toxicol 27, 519-524. doi:10.1177/0960327108092291

Manika, K., Tasiopoulou, K., Vlogiaris, L. et al. (2013). Rifampicin-associated acute renal failure and hemolysis: A rather uncommon but severe complication. Ren Fail 35, 1179-1181. doi:10.3109/0886022X.2013.815567

Matsumura, N., Takeuchi, C., Hishikawa, K. et al. (2001). Glufosinate ammonium induces convulsion through N-meth-
yl-D-aspartate receptors in mice. Neurosci Lett 304, 123-125. doi:10.1016/S0304-3940(01)01765-7

McEvoy, G. K. (2006). AHFS Drug Information 2006. $1^{\text {st }}$ edition. Bethesda Maryland, USA: American Society of Health System Pharmacists.

Meister, S., Frey, B., Lang, V. R. et al. (2010). Calcium channel blocker verapamil enhances endoplasmic reticulum stress and cell death induced by proteasome inhibition in myeloma cells. Neoplasia 12, 550-561. doi:10.1593/neo.10228

Nakada, T., Kwee, I. L., Lerner, A. M. and Remler, M. P. (1983). Theophylline-induced seizures: Clinical and pathophysiologic aspects. West J Med 138, 371-374.

Naughton, C. A. (2008). Drug-induced nephrotoxicity. Am Fam Physician 78, 743-750.

NCIt-National Cancer Institute Thesaurus (2018). Codeine Phosphate (Code C74548). https://ncit.nci.nih.gov/ncitbrowser/ ConceptReport.jsp?dictionary $=$ NCI_Thesaurus\&ns $=\mathrm{NCI}$ Thesaurus\& code $=\mathrm{C} 74548$

Nicolas, J., Hendriksen, P. J., de Haan, L. H. et al. (2015). In vitro detection of cardiotoxins or neurotoxins affecting ion channels or pumps using beating cardiomyocytes as alternative for animal testing. Toxicol In Vitro 29, 281-288. doi:10.1016/j. tiv.2014.11.010

$\mathrm{NIH}$ - National Institutes of Health (2006). Background Review Document: Validation of Neutral Red Uptake Test Methods NIH / In Vitro Cytotoxicity Test Methods for Estimating Acute Oral Systemic Toxicity. National Institutes of Health, Publication No. 07-4518. https://ntp.niehs.nih.gov/pubhealth/ evalatm/test-method-evaluations/acute-systemic-tox/invitro-validation/brd/index.html

NIH (2009). The report on the ICCVAM-NICEATM/ECVAM/ JaCVAM Scientific Workshop on Acute Chemical Safety Testing: Advancing In Vitro Approaches and Humane Endpoints for Systemic Toxicity Evaluations. https://ntp.niehs.nih.gov/ pubhealth/evalatm/3rs-meetings/past-meetings/acute-toxwksp-2008/index.html

NJ RTK - New Jersey Right to Know Hazardous Substance fact sheets (2004). 1-Naphthylamine RTK $n^{\circ}$ 1325. http://www. nj.gov/health/eoh/rtkweb/documents/fs/1325.pdf

NJ RTK (2010). Resorcinol RTK n 1634. http://www.nj.gov/ health/eoh/rtkweb/documents/fs/1634.pdf

Norlén, H., Berggren, E., Whelan, M. and Worth, A. (2012). An investigation into the use of computational and in vitro methods for acute systemic toxicity prediction. JRC Report EUR 25473 EN. Publications Office of the European Union, Luxembourg. http://publications.jrc.ec.europa.eu/repository/ handle/JRC72736

NRC - National Research Council (2015). Application of Modern Toxicology Approaches for Predicting Acute Toxicity for Chemical Defense. Washington, DC, USA: The National Academies Press. Www.nap.edu/catalog/21775/application-of-moderntoxicology-approaches-for-predicting-acute-toxicityforchemical-defense.

OECD (2016a). Guidance Document for the Use of Adverse Outcome Pathways in Developing Integrated Approaches to Testing and Assessment (IATA). Series on Testing and Assessment No. 260. OECD Publishing, Paris. http://www.oecd.org/ 
officialdocuments/publicdisplaydocumentpdf/?cote=ENV/ $\mathrm{JM} / \mathrm{MONO}(2016) 67 / \&$ doclanguage $=\mathrm{en}$

OECD (2016b). Guidance Document on the Reporting of Defined Approaches to be Used Within Integrated Approaches to Testing and Assessment. Series on Testing and Assessment No. 255. OECD Publishing, Paris. http://www.oecd.org/ officialdocuments/publicdisplaydocumentpdf/?cote $=\mathrm{env} / \mathrm{jm} /$ mono(2016)28\&doclanguage $=$ en

Olafsdóttir, K., Atzori, L., Ryrfeldt, A. et al. (1991). Mechanisms of hydroperoxide-induced broncho- and vasoconstriction in isolated and perfused rat lung. Pharmacol Toxicol 68, 181186. doi:10.1111/j.1600-0773.1991.tb01219.x

Osorio-Rico, L., Santamaria, A. and Galván-Arzate, S. (2017). Thallium toxicity: General issues, neurological symptoms, and neurotoxic mechanisms. Adv Neurobiol 18, 345-353. doi:10.1007/978-3-319-60189-2_17

Ozbek, E. (2012). Induction of oxidative stress in kidney. Int $J$ Nephrol 2012, 1-9. doi:10.1155/2012/465897

Paini, A., Joossens, E., Bessems, J. et al. (2017). EURL ECVAM workshop on new generation of physiologically-based kinetic models in risk assessment. EUR 28794 EN. Publications Office of the European Union, Luxembourg. doi:10.2760/619902

Pandey, C. K., Agarwal, A., Baronia, A. and Singh, N. (2000). Toxicity of ingested formalin and its management. Hum Exp Toxicol 19, 360-366. doi:10.1191/096032700678815954

Patel, M. N., Yim, G. K. and Isom, G. E. (1993). N-methyl-Daspartate receptors mediate cyanide-induced cytotoxicity in hippocampal cultures. Neurotoxicology 14, 35-40.

Pawar, A. T. and Vyawahare, N. S. (2017). Protective effect of ethyl acetate fraction of Biophytum sensitivum extract against sodium oxalate-induced urolithiasis in rats. $J$ Tradit Complement Med 7, 476-486. doi:10.1016/j.jtcme.2016.12.012

Peng, C., Zheng, T., Yang, F. et al. (2009). Study of neurotoxic effects and underlying mechanisms of aconitine on cerebral cortex neuron cells. Arch Pharm Res 32, 1533-1543. doi:10.1007/s12272-009-2105-1

Pershad, J., Palmisano, P. and Nichols, M. (1999). Chloral hydrate: The good and the bad. Pediatr Emerg Care 15, 432-435. doi:10.1097/00006565-199912000-00018

Persson, L. A., Norlander, B. and Kristensson, K. (1978). Studies on hexachlorophene-induced myelin lesions in the trigeminal root transitional region in developing and adult mice. Acta Neuropathol 42, 115-120. doi:10.1007/BF00690976

Pokorny, C. S. and Saunders, J. B. (1994). Codeine induced rhabdomyolysis. Aust N Z J Med 24, 582. doi:10.1111/j.14455994.1994.tb01769.x

Pomara, C., Fiore, C., D’Errico, S. et al. (2008). Calcium oxalate crystals in acute ethylene glycol poisoning: A confocal laser scanning microscope study in a fatal case. Clin Toxicol (Phila) 46, 322-324. doi:10.1080/15563650701419011

Pragst,F.,Herre, S. andBakdash,A.(2006).Poisonings with diphenhydramine-A survey of 68 clinical and 55 death cases. Forensic Sci Int 161, 189-197. doi:10.1016/j.forsciint.2006.01.019

Prassas, I., Karagiannis, G. S., Batruch, I. et al. (2011). Digitoxin-induced cytotoxicity in cancer cells is mediated through distinct kinase and interferon signaling networks. Mol Cancer Ther 10, 2083-2093. doi:10.1158/1535-7163.MCT-11-0421
Prieto, P., Kinsner-Ovaskainen, A., Stanzel, S. et al. (2013a). The value of selected in vitro and in silico methods to predict acute oral toxicity in a regulatory context: Results from the European Project ACuteTox. Toxicol In Vitro 27, 357-376. doi:10.1016/j.tiv.2012.07.013

Prieto, P., Cole, T., Curren, R. et al. (2013b). Assessment of the predictive capacity of the $3 \mathrm{~T} 3$ neutral red uptake cytotoxicity test method to identify substances not classified for acute oral toxicity (LD50 > $2000 \mathrm{mg} / \mathrm{kg}$ ): Results of an ECVAM validation study. Regul Toxicol Pharmacol 65, 344-365. doi:10.1016/j.yrtph.2012.11.013

Qiu, J., Gao, H.Q., Liang, Y. et al. (2008). Comparative proteomics analysis reveals role of heat shock protein 60 in digoxin-induced toxicity in human endothelial cells. Biochim Biophys Acta 1784, 1857-1864. doi:10.1016/j.bbapap.2008.07.006

Raudenska, M., Gumulec, J., Babula, P. et al. (2013). Haloperidol cytotoxicity and its relation to oxidative stress. Mini Rev Med Chem 13, 1993-1998. doi:10.2174/13895575113136660 100

Rejdak, K., Nieoczym, D., Czuczwar, M. et al. (2011). Orphenadrine induces secondarily generalized convulsive status epilepticus in rats. Brain Res Bull 84, 389-393. doi:10.1016/j. brainresbull.2011.01.014

Rho, J. M., Donevan, S. D. and Rogawski, M. A. (1997). Barbiturate-like actions of the propanediol dicarbamates felbamate and meprobamate. J Pharmacol Exp Ther 280, 1383-1391.

Riyaz, R., Pandalai, S. L., Schwartz, M. and Kazzi, Z. N. (2013). A fatal case of thallium toxicity: Challenges in management. $J$ Med Toxicol 9, 75-78. doi:10.1007/s13181-012-0251-1

Robinson, S., Delongeas, J. L., Donald, E. et al. (2008). A European pharmaceutical company initiative challenging the regulatory requirement for acute toxicity studies in pharmaceutical drug development. Regul Toxicol Pharmacol 50, 345-352. doi:10.1016/j.yrtph.2007.11.009

Sabeti, J., Gerhardt, G. A. and Zahniser, N. R. (2003). Chloral hydrate and ethanol, but not urethane, alter the clearance of exogenous dopamine recorded by chronoamperometry in striatum of unrestrained rats. Neurosci Lett 343, 9-12. doi:10.1016/ S0304-3940(03)00301-X

Sachana, M., Flaskos, J., Nikolaidis, E. et al. (2001). Inhibition of rat platelet 5-hydroxytryptamine uptake by chlorpyrifos and carbaryl. Pharmacol Toxicol 89, 195-200. doi:10.1111/j.09019928.2001.890409.x

Sachana, M. and Leinala, E. (2017). Approaching chemical safety assessment through application of integrated approaches to testing and assessment: Combining mechanistic information derived from adverse outcome pathways and alternative methods. Appl In Vitro Toxicol 3, 227-233. doi:10.1089/aivt. 2017.0013

Safirstein, R. L. (2004). Acute renal failure: from renal physiology to the renal transcriptome. Kidney Int, Suppl 91, S62-66. doi:10.1111/j.1523-1755.2004.09110.x

Sangster, B., van Heijst, A. N. and Zimmerman, A. N. (1978). (Orphenadrine poisoning (Disipal)). Ned Tijdschr Geneeskd 122, 988-992.

Saukkonen, J. J., Cohn, D. L., Jasmer, R. M. et al. (2006). An official ATS statement: Hepatotoxicity of antituberculosis ther- 
apy. Am J Respir Crit Care Med 174, 935-952. doi:10.1164/ rccm.200510-1666ST

SCHEER - Scientific Committee on Health, Environmental and EmergingRisks(2018). Memorandumonweightofevidenceand uncertainties. Opinion adopted 26 June 2018. Scientific Committee on Health, Environmental and Emerging Risks. https:// ec.europa.eu/health/sites/health/files/scientific_committees/ scheer/docs/scheer_o_014.pdf

Schep, L. J., Slaughter, R. J., Temple, W. A. and Beasley, D. M. (2009). Diethylene glycol poisoning. Clin Toxicol 47, 525535. doi:10.1080/15563650903086444

Scott, A. M., Powell, G. M., Upshall, D. G. and Curtis, C. G. (1990). Pulmonary toxicity of thioureas in the rat. Environ Health Perspect 85, 43-50. doi:10.2307/3430664

Seidle, T., Robinson, S., Holmes, T. et al. (2010). Cross-sector review of drivers and available 3Rs approaches for acute systemic toxicity testing. Toxicol Sci 116, 382-396. doi:10.1093/ toxsci/kfq143

Simoneschi, D., Simoneschi, F. and Todd, N. E. (2014). Assessment of cardiotoxicity and effects of malathion on the early development of zebrafish (Danio Rerio) using computer vision for heart rate quantification. Zebrafish 11, 275-280. doi:10.1089/zeb.2014.0973

Spencer, C. I., Yuill, K. H., Borg, J. J. et al. (2001). Actions of pyrethroid insecticides on sodium currents, action potentials, and contractile rhythm in isolated mammalian ventricular myocytes and perfused hearts. J Pharmacol Exp Ther 298, 1067-1082.

Stokes, W. S., Casati, S., Strickland, J. and Paris, M. (2008). Neutral red uptake cytotoxicity tests for estimating starting doses for acute oral toxicity tests. Curr Protoc Toxicol, Unit 20.4, 1-20. doi:10.1002/0471140856.tx2004s36

Strickland, J., Clippinger, A. J., Brown, J. et al. (2018). Status of acute systemic toxicity testing requirements and data uses by U.S. regulatory agencies. Regul Toxicol Pharmacol 3, 183196. doi:10.1016/j.yrtph.2018.01.022

Sun, G. B., Sun, H., Meng, X. B. et al. (2014). Aconitine-induced $\mathrm{Ca}^{2+}$ overload causes arrhythmia and triggers apoptosis through p38 MAPK signaling pathway in rats. Toxicol Appl Pharmacol 279, 8-22. doi:10.1016/j.taap.2014.05.005

Sztajnkrycer, M. D. (2002). Valproic acid toxicity: overview and management. J Toxicol Clin Toxicol 40, 789-801. doi:10.1081/ CLT-120014645

Takahama, K. and Shirasaki, T. (2007). Central and peripheral mechanisms of narcotic antitussives: Codeine-sensitive and -resistant coughs. Cough 3, 8. doi:10.1186/1745-9974-3-8

Teske, J., Weller, J. P., Albrecht, U. V. and Fieguth, A. (2011). Fatal intoxication due to brucine. J Anal Toxicol 35, 248-253. doi:10.1093/anatox/35.4.248

TOXNET - Toxicology data network (2015). Valproic acid. https://toxnet.nlm.nih.gov/cgi-bin/sis/search/a?dbs+hsdb:@ term+@DOCNO+3582 (accessed 09.07.2018).

Vinken, M. and Blaauboer, B. J. (2017). In vitro testing of basal cytotoxicity: Establishment of an adverse outcome pathway from chemical insult to cell death. Toxicol In Vitro 39, 104110. doi:10.1016/j.tiv.2016.12.004

Vos, J. G., van Logten, M. J., Kreeftenberg, J. G. and Kruizinga,
W. (1984). Effect of triphenyltin hydroxide on the immune system of the rat. Toxicology 29, 325-336. doi:10.1016/0300483X(84)90164-1

Vučević, D., Petronijević, N., Radonjić, N. et al. (2009). Acetylcholinesterase as a potential target of acute neurotoxic effects of lindane in rats. Gen Physiol Biophys, Spec Issue 28, 18-24.

Wan, L. and O'Brien, P. (2014). Molecular mechanism of $17 \alpha$-ethinylestradiol cytotoxicity in isolated rat hepatocytes. Can J Physiol Pharmacol 92, 21-26. doi:10.1139/cjpp-20130267

Williams, M. and Robinson, J. L. (1984). Nicotinic receptors in mammalian brain. Prog Neuropsychopharmacol Biol Psychiatry 8, 769-772. doi:10.1016/0278-5846(84)90056-3

Williamson, D., Winterbourn, C. C., Swallow, W. H. and Missen, A. W. (1981). Oxidative hemoglobin breakdown induced by a rubber additive. Hemoglobin 5, 73-84. doi:10.3109/ 03630268108996911

Wood, D. (2014). Formaldehyde and metaldehyde. In $\mathrm{N}$. Bateman, R. Jefferson, S. Thomas et al. (eds.), Oxford Desk Reference Toxicology (245-248). $1^{\text {st }}$ edition. Oxford, UK: Oxford University Press.

Woolf, A. D., Erdman, A. R., Nelson, L. S. et al. (2007). Tricyclic antidepressant poisoning: An evidence-based consensus guideline for out-of-hospital management. Clin Toxicol (Phila) 45, 203-233. doi:10.1080/15563650701226192

Xiong, J., Zhang, X., Huang, J. et al. (2016). Fenpropathrin, a widely used pesticide, causes dopaminergic degeneration. Mol Neurobiol 53, 995-1008. doi:10.1007/s12035-014-9057-2

Yuen, H. W. and Gossman, W. G. (2018). Iron Toxicity. In StatPearls [Internet]. Treasure Island (FL): StatPearls Publishing. https://www.ncbi.nlm.nih.gov/books/NBK459224/

Zaldivar Comenges, J. M., Joossens, E., Benito, J. V. S. et al. (2017). Theoretical and mathematical foundation of the virtual cell based assay - A review. Toxicol In Vitro 45, 209-221. doi:10.1016/j.tiv.2016.07.013

Zbinden, G. and Flury-Roversi, M. (1981). Significance of the LD50-test for the toxicological evaluation of chemical substances. Arch Toxicol 47, 77-99. doi:10.1007/BF00332351

Zhang, W., Shibamoto, T., Kuda, Y. et al. (2011). Pulmonary vasoconstrictive and bronchoconstrictive responses to anaphylaxis are weakened via $\beta 2$-adrenoceptor activation by endogenous epinephrine in anesthetized rats. Anesthesiology 114, 614-623. doi:10.1097/ALN.0b013e31820b8d34

Zhou, Y., Vaidya, V. S., Brown, R. P. et al. (2008). Comparison of kidney injury molecule-1 and other nephrotoxicity biomarkers in urine and kidney following acute exposure to gentamicin, mercury, and chromium. Toxicol Sci 101, 159-170. doi: $10.1093 /$ toxsci/kfm260

Zorn, K. E. and Fudin, J. (2011). Treatment of neuropathic pain: The role of unique opioid agents. Practical Pain Management. http://paindr.com/wp-content/uploads/2012/06/OpioidNerve-Pain_Fudin-ZORN.pdf

\section{Conflict of interest}

The authors declare that they have no conflict of interest to disclose. 